\title{
A statistically significant lack of debris discs in medium separation binary systems
}

\author{
Ben Yelverton, ${ }^{1 \star}$ Grant M. Kennedy ${ }^{\odot}, 2,3$ Kate Y. L. Su${ }^{4}$ and Mark C. Wyatt ${ }^{\oplus 1}$ \\ ${ }^{1}$ Institute of Astronomy, University of Cambridge, Madingley Road, Cambridge CB3 OHA, UK \\ ${ }^{2}$ Department of Physics, University of Warwick, Gibbet Hill Road, Coventry CV4 7AL, UK \\ ${ }^{3}$ Centre for Exoplanets and Habitability, University of Warwick, Gibbet Hill Road, Coventry CV4 7AL, UK \\ ${ }^{4}$ Steward Observatory, University of Arizona, 933 N Cherry Avenue, Tucson, AZ 85721, USA
}

Accepted 2019 July 9. Received 2019 July 8; in original form 2019 May 23

\begin{abstract}
We compile a sample of 341 binary and multiple star systems with the aim of searching for and characterizing Kuiper belt-like debris discs. The sample is assembled by combining several smaller samples studied in previously published work with targets from two unpublished Herschel surveys. We find that 38 systems show excess emission at 70 or $100 \mu \mathrm{m}$ suggestive of a debris disc. While nine of the discs appear to be unstable to perturbations from their host binary based on a simple analysis of their inferred radii, we argue that the evidence for genuine instability is not strong, primarily because of uncertainty in the true disc radii, uncertainty in the boundaries of the unstable regions, and orbital projection effects. The binary separation distributions of the disc-bearing and disc-free systems are different at a confidence level of 99.4 per cent, indicating that binary separation strongly influences the presence of detectable levels of debris. No discs are detected for separations between $\sim 25$ and $135 \mathrm{au}$; this is likely a result of binaries whose separations are comparable with typical disc radii clearing out their primordial circumstellar or circumbinary material via dynamical perturbations. The disc detection rate is $19_{-3}^{+5}$ per cent for binaries wider than $135 \mathrm{au}$, similar to the published results for single stars. Only $8_{-1}^{+2}$ per cent of systems with separations below 25 au host a detectable disc, which may suggest that planetesimal formation is inhibited in binaries closer than a few tens of au, similar to the conclusions of studies of known planet-hosting binaries.
\end{abstract}

Key words: binaries: general-circumstellar matter.

\section{INTRODUCTION}

The planet formation process, characterized by the accumulation of micron-sized dust particles in the protoplanetary disc into increasingly large bodies, produces a population of planetesimals with sizes of order 1-1000 km (Johansen et al. 2014). The primordial dust is expected to be removed from the disc by Poynting-Robertson (PR) drag within a few Myr (Wyatt et al. 2015), leaving behind these larger bodies. Yet, a significant proportion of stars older than $\sim 10 \mathrm{Myr}$ show excess flux at infrared wavelengths, which is generally interpreted as thermal emission from circumstellar dust that has been heated by the stellar radiation (e.g. Aumann et al. 1984; Oudmaijer et al. 1992; Mannings \& Barlow 1998). Rather than being primordial, the dust responsible for this emission is understood to be continuously replenished by destructive collisions between the planetesimals (Backman \& Paresce 1993; Dominik \& Decin 2003).

\footnotetext{
^E-mail: bmy21@cam.ac.uk
}

These discs of planetesimals and the products of their collisions are known as debris discs, and can be distinguished observationally from primordial dust discs by their relatively low fractional luminosities (e.g. Hughes, Duchêne \& Matthews 2018). Importantly, an infrared excess corresponding to a debris disc in a given system acts as a signpost that the process of planet formation was able to proceed to a significant degree in that system. The proportion of stars with detected debris discs has been estimated at around 25 percent for A- and F-type stars, and close to 15 per cent for $\mathrm{G}$ and $\mathrm{K}$ types (Su et al. 2006; Hillenbrand et al. 2008; Trilling et al. 2008; Eiroa et al. 2013; Sierchio et al. 2014; Thureau et al. 2014; Sibthorpe et al. 2018).

Binary and higher order multiple stars make up a large fraction of all known star systems; for example, Raghavan et al. (2010) estimate that 44 per cent of FGK stars have at least one stellar companion (see also Duquennoy \& Mayor 1991; Eggleton \& Tokovinin 2008; Duchêne \& Kraus 2013). Planets do not appear to be uncommon in such systems, with Horch et al. (2014) concluding that $\sim 40$ 50 per cent of Kepler planet hosts are binaries, comparable with the binary fraction of field stars. Similarly, Armstrong et al. (2014) used 
Kepler data to infer that the occurrence rate of circumbinary planets with radii above $6 R_{\oplus}$ and periods less than $300 \mathrm{~d}$ is consistent with single star rates. However, the planet occurrence rate is strongly dependent on stellar separation. For example, Kraus et al. (2016) estimated that binaries closer than $\sim 50$ au are around a third as likely as wider systems to host a planet. Thus, it is important to consider the problem of how planetary systems form and evolve around binary systems. Knowledge of the properties of debris discs in binary systems, and how they depend on the stellar orbits, can contribute to our understanding of how often, and where, we expect planetesimals to be able to form therein, with implications for planets.

Several previous works have addressed this issue. Trilling et al. (2007) observed 69 AFGK binary and multiple systems with the Multiband Imaging Photometer for Spitzer (MIPS; Rieke et al. 2004) to search for excess emission at 24 and $70 \mu \mathrm{m}$. Considering as 'excesses' measurements at least $2 \sigma$ above the expected stellar flux, they found a rather high overall disc detection fraction of around 40 per cent. Their results also suggested that binaries with orbital separations of 3-50 au were less likely to host a disc than those with closer or wider separations, with the explanation that such binaries have separations comparable to typical debris disc radii, such that planetesimals in those systems would tend to be in an unstable location. None the less, three of their excesses implied the presence of dust in dynamically unstable regions (Holman \& Wiegert 1999), and they proposed that these could be explained as dust migrating inwards from a stable region due to PR drag. However, this mechanism likely cannot provide a general explanation for detectable levels of apparently unstable dust, as dust grains in detectable debris discs are expected to be removed via collisional evolution and radiation pressure on time-scales faster than PR drag can act (Wyatt 2005).

Rodriguez \& Zuckerman (2012) approached the problem differently, starting by gathering a sample of 112 (largely AFGK) stars thought to host discs based on previous work, and found that 28 of them belonged to a binary or multiple system. The distribution of separations of the binaries in their sample showed fewer systems in the 1-100 au range than would be expected for a randomly selected sample, again suggesting that 'medium' separation binaries are less likely to host discs.

The most extensive study of debris discs in binary and multiple systems performed to date has been that of Rodriguez et al. (2015). They started with a volume-limited sample of 449 AFGKM stars observed by Herschel as part of the DEBRIS programme (Matthews et al. 2010), of which they identified 188 as belonging to a binary or multiple system. Combining 100 and $160 \mu \mathrm{m}$ photometry from Herschel's Photodetector Array Camera and Spectrometer (PACS; Poglitsch et al. 2010) with archival photometry at other mid to far-infrared wavelengths, and using a $3 \sigma$ excess criterion, they found a low overall disc detection rate of around 11 percent for the binaries and multiples versus 21 percent for the single stars in their sample, in contrast to the results of Trilling et al. (2007). Superficially, their results suggested that binaries with periods of around $10^{3}-10^{5} \mathrm{~d}$ (corresponding to semimajor axes of around 150 au for Sun-like stars, which make up the majority of their sample) are less likely to host debris discs, similar to the conclusions of previous work. However, using a Kolmogorov-Smirnov (KS) test, they demonstrated that the period distributions of their disc-bearing and disc-free binaries and multiples were not, in fact, different in a statistically significant way (with a $p$-value of 0.09 ).

In this paper, we compile a large sample of binary and multiple systems that have photometric measurements in the far-infrared, comprising systems drawn from several sources. Our sample con- sists of most of the systems analysed by Trilling et al. (2007), Rodriguez \& Zuckerman (2012), and Rodriguez et al. (2015), with the addition of systems from two unpublished Herschel surveys: OT2_gkennedy_2, which targeted visual binaries with well-known orbits, and OT1_jdrake01_1, which targeted close binaries. Considering overlap between the various sources, there are 341 unique systems in the full sample. We aim to use this sample to quantify the incidence and properties of debris discs in binary and multiple systems with higher statistical significance than has been possible in previous work.

We expect such a result to be possible first because we simply have a larger sample, and secondly because of the inclusion of the OT2_gkennedy_2 systems in particular. As they have well known orbits, their semimajor axes are in the $1-50$ au range (for wider binaries, observations spanning a very long time period would be required to deduce their orbits precisely). Thus, these systems lie in the separation range of greatest interest, where previous work suggests that the separation distributions of disc-bearing and discfree systems may differ. The inclusion of the OT2_gkennedy_2 data also allows us to examine separately those systems whose orbits are well known.

For our analysis, we gather archival photometry for each system to create spectral energy distributions (SEDs), then model these in search of $3 \sigma$ excesses in Spitzer $70 \mu \mathrm{m}$ and Herschel 70 and $100 \mu \mathrm{m}$ photometry characteristic of cool debris. Although broadly similar SED modelling of many of our systems has been performed in previous studies, analysing our sample as a whole rather than relying on the results of previous work removes any issues of inconsistency - for example, Trilling et al. (2007) used $2 \sigma$ as their excess threshold, while Rodriguez et al. (2015) used $3 \sigma$.

In Section 2, we describe in detail the make-up of our sample. Section 3 explains our methods for searching for and characterizing debris discs within the sample using SEDs. The results of this analysis, with a particular emphasis on disc stability and detection statistics, are presented in Section 4. In Section 5, we consider how our results relate to those of studies of protoplanetary discs and planets in binaries, then discuss what can be learnt about close binaries from the OT1_jdrake01_1 data. We present our overall conclusions in Section 6.

\section{THE SAMPLE}

This section explains how our sample of binary and multiple star systems was compiled, before briefly presenting the orbital and stellar properties of the sample as a whole. As the aim of this paper is to search for and characterize excesses in the far-infrared, where debris disc emission typically peaks, we compile systems that have been observed at $70 \mu \mathrm{m}$ by MIPS or at 70 and/or $100 \mu \mathrm{m}$ by PACS.

First, we include all 34 systems targeted by the Herschel survey OT2_gkennedy_2. All were observed at 70 and $160 \mu \mathrm{m}$ by PACS. The bulk of these systems were drawn from the Sixth Catalog of Orbits of Visual Binary Stars (VB6; Hartkopf, Mason \& Worley 2001). This survey aimed to obtain observations of binaries whose orbits are well known, and so systems with highly graded orbital solutions in VB6 (grade 1, 'definitive', or grade 2, 'good') were chosen. The list of targets was narrowed by retaining only those stars with spectral types AFG and luminosity classes V or IV$\mathrm{V}$, and within a distance of $40 \mathrm{pc}$. Further exclusions were made of stars with non-main-sequence positions on a HertzsprungRussell diagram, and one system lying in the Galactic plane. In addition, we excluded systems with existing far-infrared observa- 
tions: several with PACS observations from other programmes, one (HD 26690) with a MIPS $70 \mu \mathrm{m}$ observation from Trilling et al. (2007) suggesting a lack of dust, and two (HD 40183 and HD $155125)$ with constraints from the Infrared Astronomical Satellite (IRAS).

The resulting sample contains 27 systems. Of the remaining seven targets in the programme, three (HD 46273, HD 80671, and HD 127726) are those systems suggested by Trilling et al. (2007) to harbour unstable dust, and were included with the aim of resolving the proposed discs. The final four (HD 31925, HD 95698, HD 173608, and HD 217792) are systems with dust temperatures that were poorly constrained based on the data of Trilling et al. (2007), and were included to allow us to better characterize their possible excesses with the addition of $160 \mu \mathrm{m}$ photometry.

To these, we add the 51 systems observed at 70 and $160 \mu \mathrm{m}$ by PACS as part of the Herschel survey OT1_jdrake01_1. This survey was motivated by the work of Matranga et al. (2010), which suggested that $\sim 20-30$ per cent of close binaries host large amounts of very warm dust (with temperatures above $\sim 1000 \mathrm{~K}$ ), and interpreted this dust as a possible product of collisions between planetary bodies that have been destabilised by long-term changes in the binaries' orbits. The OT1_jdrake01_1 survey was designed to be complementary to that work: it aimed to search for cooler dust components around close binaries in order to build a more complete picture of their typical planetary systems (Drake 2010). As this cooler dust is indicative of a more typical debris disc scenario in which ongoing collisions between planetesimals replenish the dust - and to our knowledge there exists no published analysis of these data - we include these systems in our sample.

We also include almost all of the binary and multiple systems studied by Trilling et al. (2007), Rodriguez \& Zuckerman (2012), and Rodriguez et al. (2015). The total numbers of binaries and multiples considered in each of those works are 69, 28, and 188, respectively. There is some overlap between the various samples: 15 systems appear in two of the previously mentioned studies, and two systems appear in all three. Additionally, nine systems from OT2_gkennedy_2 and one from OT1_jdrake01_1 (HD 118216) are in the Trilling et al. (2007) sample. There is also the special case of HD 131976 (in OT1_jdrake01_1) and HD 131977 (in Rodriguez et al. 2015): the latter is the primary star of the system GJ 570, while the former is the secondary component, itself a spectroscopic binary (see e.g. VB6). Since for most binary and multiple systems we do not have separate far-infrared observations of each component, for consistency we discard the secondary, HD 131976, from our sample; for completeness, we note that its SED does not show an infrared excess. We exclude also six systems from Rodriguez \& Zuckerman (2012) - HD 1051, HD 8538, HD 125473, HD 138749, HD 150378, and HD 169022 - as these only appear to have been observed in the far-infrared by IRAS. Owing to the low spatial resolution of IRAS, its photometry is prone to contamination from background sources, so that any apparent IRAS excess without confirmation by MIPS or PACS would be somewhat less believable. Further two systems are excluded from Trilling et al. (2007) - HD 61497 and HD 111066 - since they were observed only at $24 \mu \mathrm{m}$ during the survey presented in that paper and have not since been followed up at longer wavelengths by either MIPS or PACS.

Finally, we add the following nine systems with highly graded VB6 orbits to the sample. HD 128620 was observed as part of the DEBRIS survey, and HD 48915 as part of Herschel calibration; both would have been targeted by OT2_gkennedy_2 if not for these existing observations. ${ }^{1}$ The systems HD 15285, HD 79969, HD 115953, HD 148653, HD 165341, HD 184467, and HD 196795 were observed by PACS as part of the DUNES survey (Eiroa et al. 2010). All are of spectral type $\mathrm{K}$ or $\mathrm{M}$, and therefore despite their wellknown orbits, would not have been included in OT2_gkennedy_2 even if they had not been targeted by DUNES. None the less, as we are not requiring that our whole sample be composed only of AFG stars, we include them in our analysis.

The resulting full sample contains 341 unique systems. The stars in the sample are largely primaries, but two of them are secondaries. The first is HD 137392, which was targeted by OT2_gkennedy_2 instead of its primary HD 137391 because the $\mathrm{BaBb}$ orbit of that system, but not the AB orbit, is highly graded in VB6. The second is HD 14082B, which was included in the sample of Rodriguez \& Zuckerman (2012) since it is known that the secondary hosts a detectable disc while the primary HD 14082A does not. We note that an alternative approach to constructing a sample of binaries would be to use Gaia Data Release 2 (Gaia Collaboration 2018) astrometry to make a list of pairs of stars with common proper motion. However, for this study we require stars that have been observed in the far-infrared, which would not generally be the case for the proposed Gaia sample. Conversely, for those systems that do not have well-characterized orbits - which constitute the majority of the sample, as discussed next - one might hope to use the Gaia proper motions to confirm or refute their binary nature. We do not, however, attempt to do so since the stars we will study are generally bright, with over half of the sample having a $V$-band magnitude below 6, where Gaia astrometry can be unreliable (Lindegren et al. 2018).

As we are interested in how the presence of debris depends on the stellar orbits, for each system in our sample we record the stellar separations $a$ and, where available, orbital eccentricities $e$; this information is presented in Table 1. Systems containing three or more stars have two or more separations. For the systems from Trilling et al. (2007), Rodriguez \& Zuckerman (2012), and Rodriguez et al. (2015), we mostly use the values given in those papers; their separations are not necessarily true semimajor axes, but in many cases projected separations. Of these systems, for those that appear in the VB6 catalogue with grade 1 or 2 we preferentially use the VB6 values - the separations of these systems are true semimajor axes and can be considered well known. In some cases, the orbits given in the paper from which we took a system are incomplete; where possible, we supplement these with VB6 elements, even if they are poorly graded. For example, the triple system HD 196885 is listed in Trilling et al. (2007) as having a separation of 190 arcsec. This is for the orbit of the AB pair, and VB6 gives an additional grade 5 orbit for the AaAb pair with a separation of 0.64 arcsec, which we record for completeness. In addition, HD 181296 is listed with only one separation (199 au) in Rodriguez \& Zuckerman (2012). This is in fact a triple system, with the more distant companion being HD 181327, and as the system does not appear in VB6 we take the wider separation $\left(\sim 2 \times 10^{4} \mathrm{au}\right)$ from Tokovinin (2018).

To convert the VB6 angular separations into physical separations, we mainly use parallaxes from Hipparcos (van Leeuwen 2007). ${ }^{2}$

\footnotetext{
${ }^{1}$ Since we ultimately chose to focus on MIPS and PACS data, we do not include in our study the two systems that were excluded from OT2_gkennedy_2 because of their existing IRAS photometry.

${ }^{2}$ For systems not in the Hipparcos catalogue, we mostly use parallaxes from Gaia Data Release 2 (Gaia Collaboration 2018). For the few systems that appear in neither catalogue, we source parallaxes from Harrington \& Dahn
} 
Table 1. Information on the orbits of the binaries and multiple systems in our sample. The columns from left to right show the name of each system; its 'source', i.e. the paper or programme from which we took the system (see text of Section 2 for details); its distance, calculated as the reciprocal of the parallax; the names of the components that the given orbits refer to; the VB6 orbit grades, semimajor axes, and eccentricities where available; and the separations and eccentricities given in the source where available. For systems from the OT1_j drake01_1 programme, the 'source' separations are the values that we estimated from their periods, as detailed in Section 2. Here, we display a 10-line excerpt chosen to show systems from a variety of sources; the full 341-line table is available online in machine-readable form.

\begin{tabular}{|c|c|c|c|c|c|c|c|c|c|}
\hline Name & Source & Distance / pc & Component & Grade & VB6 a/arcsec & $\operatorname{VB} 6 a / \mathrm{au}$ & VB6e & Source $a$ / au & Source $e$ \\
\hline & & & & $\cdots$ & & & & & \\
\hline HD 81858 & Kennedy & 33.2 & AB & 2 & 0.8599 & 28.52 & 0.5619 & - & - \\
\hline HD 82328 & Rodriguez15 & 13.5 & $\mathrm{AB}$ & - & - & - & - & 55.3 & - \\
\hline HD 82434 & Kennedy & 18.8 & $\mathrm{AB}$ & 1 & 0.81 & 15.24 & 0.431 & - & - \\
\hline HD 82885 & Rodriguez15 & 11.4 & $\mathrm{AB}$ & 5 & 3.84 & 43.66 & 0.88 & 43.6 & 0.88 \\
\hline HD 83808 & Trilling07 & 40.0 & $\mathrm{AaAb}$ & 2 & 0.00446 & 0.18 & 0 & 0.19 & - \\
\hline HD 86146 & Drake & 28.8 & $\mathrm{AB}$ & - & - & - & - & 0.103 & - \\
\hline HD 88215 & Trilling07 & 27.7 & $\mathrm{AB}$ & - & - & - & - & 0.2 & - \\
\hline HD 89125 & Rodriguez15 & 22.8 & $\mathrm{AB}$ & - & - & - & - & 168.6 & - \\
\hline
\end{tabular}

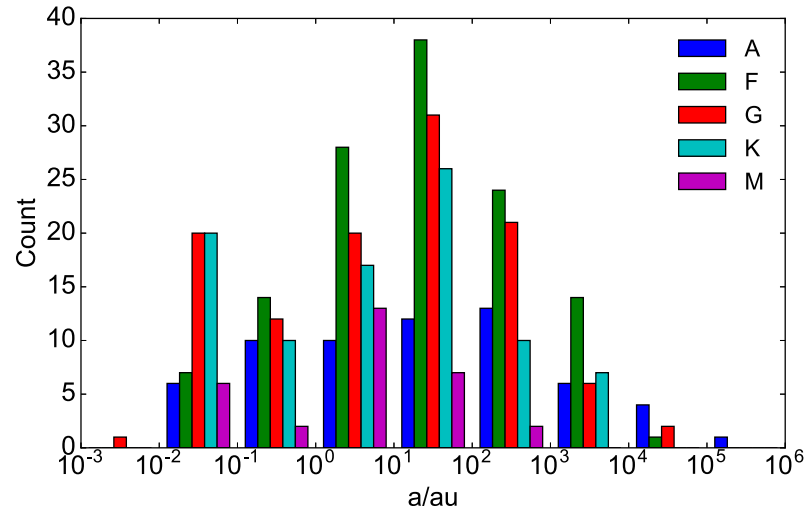

Figure 1. Distribution of stellar separations in our sample, binned by order of magnitude in separation and split by spectral type. Though there is some variation in the proportions of each spectral type between bins, the sample is largely Sun-like across the full range of separations.

For the OT2_gkennedy_2 systems - other than those that were re-observations of the Trilling et al. (2007) survey - we use VB6 orbital elements since by construction they are highly graded. For the OT1_jdrake01_1 systems, we first find orbital periods, mostly from the Ninth Catalogue of Spectroscopic Binary Orbits (SB9; Pourbaix et al. 2004). ${ }^{3}$ We estimate their luminosities $L$ from SED fitting (see Section 3 for details), calculate approximate masses $M$ assuming $L \propto M^{3.5}$, then use these to estimate stellar separations from the periods.

The make-up of our sample is outlined in Fig. 1, which shows the distribution of stellar separations for each spectral type. The spectral types are derived by binning the stars by their effective temperature $T$ from SED fitting (again, see Section 3 for details), with $T>7500 \mathrm{~K}$ classified as type A, $6000 \mathrm{~K}<T<7500 \mathrm{~K}$ as F, $5200 \mathrm{~K}<T<6000 \mathrm{~K}$ as $\mathrm{G}, 3700 \mathrm{~K}<T<5200 \mathrm{~K}$ as $\mathrm{K}$, and $T<$

(1980); Geyer, Harrington \& Worley (1988); van Altena, Lee \& Hoffleit (1995); Costa et al. (2005); Henry et al. (2006); and Torres, Andersen \& Giménez (2010).

${ }^{3}$ For the seven systems not listed in SB9, we source periods from the Multiple Star Catalog (Tokovinin 2018), Balona (1987), Frasca et al. (2006), and Kiraga (2012).
$3700 \mathrm{~K}$ as M. Systems of three or more stars, and thus two or more separations, are counted multiple times. Note that the bimodality of the distribution is not representative of binary and multiple systems generally, but is largely the result of having included a significant number of binaries (from OT1_jdrake01_1) targeted specifically because they are close.

Given that stellar ages are typically highly uncertain, in this paper we do not attempt to study disc properties as a function of age. As none of the samples from which we assembled our overall sample were selected based on age, in what follows we assume that stars of each spectral type have ages uniformly distributed up to the typical main-sequence lifetime of that type. Fig. 1 shows that the sample is dominated by FGK stars, so we expect the average age of the systems we are studying to be a few Gyr.

To summarize, we have assembled a sample of 341 binary and higher order star systems and compiled information on their stellar separations; in most cases these are projected separations taken from the literature. We define a 'known orbit' subsample consisting of all systems with at least one highly graded VB6 orbit and whose separations are therefore true semimajor axes; there are 89 such systems. For statistical analysis, care must be taken to avoid bias with respect to disc detection. Since Rodriguez \& Zuckerman (2012) studied only systems already thought to host discs, we will also define a 'statistical' subsample consisting of all systems except those taken from that paper. This subsample contains 328 systems (see Section 4.4).

Having established the systems to be studied in this paper, in the following section we outline our analysis of their SEDs and how we use these to determine whether a debris disc is present.

\section{SED MODELLING}

Our initial aim is to establish whether or not each system in our sample hosts a detectable debris disc. This is equivalent to asking whether its SED indicates an excess of flux at infrared wavelengths, beyond what would be expected for a purely stellar source. To answer this, we first gather archival photometry for each system to create SEDs. The photometry available varies between systems, but all SEDs have a range of points spanning visible to far-infrared wavelengths. By construction of the sample, all have an MIPS $70 \mu \mathrm{m}$, PACS $70 \mu \mathrm{m}$, and/or PACS $100 \mu \mathrm{m}$ observation; 
Table 2. Photometry in the MIPS $70 \mu \mathrm{m}$, PACS $70 \mu \mathrm{m}$, and PACS $100 \mu \mathrm{m}$ bands for all systems in our sample. For each band, the columns show the observed flux density, its associated uncertainty, the predicted photospheric flux density, and the significance as defined in equation (1). For consistency, here we display the data for the same 10 systems as in Table 1; the full 341-line table is available online in machine-readable form.

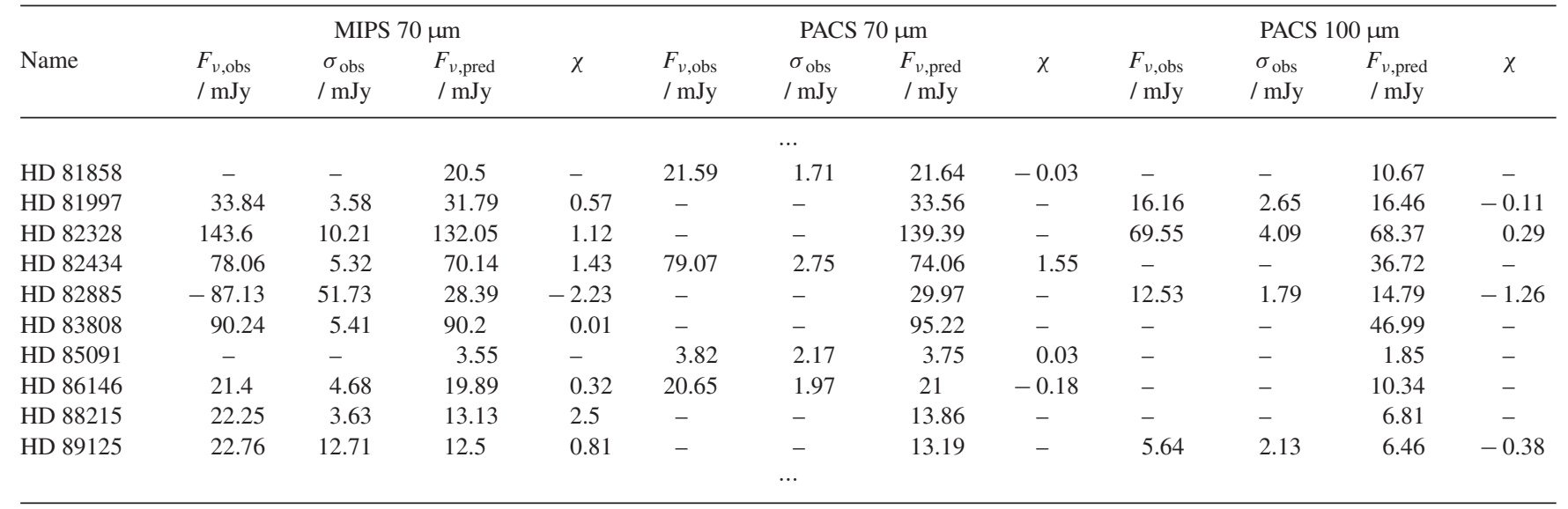

Table 2 shows the photometry we use in these bands. For PACS photometry, we use our own flux measurements mostly from point spread function (PSF) fitting, and from aperture photometry for systems with resolved discs (such as HD 95698, as discussed in appendix A), as outlined in, e.g. Sibthorpe et al. (2018).

For MIPS, we use photometry from the literature in some cases, but for most systems we make our own updated flux measurements. The general procedure is outlined in Sierchio et al. (2014), where PSF photometry is used for unresolved sources. Since most of the MIPS observations were taken at both 24 and $70 \mu \mathrm{m}$, we generally use the MIPS $24 \mu \mathrm{m}$ data to guide the position of the $70 \mu \mathrm{m}$ detection. For sources not detected at $70 \mu \mathrm{m}$, we place an aperture at the $24 \mu \mathrm{m}$ position to estimate the flux. As a result, such sources can have negative flux due to complex background structure and instrumental artefacts. Due to the large beam size of the MIPS $70 \mu \mathrm{m}$ channel, in some cases only one MIPS $70 \mu \mathrm{m}$ flux is extracted even if there are two MIPS $24 \mu \mathrm{m}$ sources near the same position.

To each SED, we then fit a model with both a stellar and a dust component, using the MULTINEST algorithm (Feroz, Hobson \& Bridges 2009). ${ }^{4}$ For the stellar component, we use PHOENIX BT-Settl models (Allard, Homeier \& Freytag 2012) with stellar abundances from Asplund et al. (2009), as these are available over a wide range of parameters. Although some binaries will be sufficiently close that both stars contribute to the total measured flux, in all cases we fit only a single stellar component. We do not expect this simplification to affect our results significantly because if the stars have different spectral types then the primary star will dominate the emission so that the secondary can be ignored. If both stars are of the same spectral type and thus both contribute significantly to the total flux, then the overall spectrum will be well approximated by a single star of the same spectral type. In addition, most systems have multiple mid-infrared flux measurements, meaning that the Rayleigh-Jeans tail - the part of the stellar spectrum that will be compared with the measured far-infrared flux to search for an excess - will be well constrained regardless of the details of the stellar model. We model the dust as a modified blackbody - i.e. its emission is given by a blackbody function multiplied by a factor $\left(\lambda_{0} / \lambda\right)^{\beta}$ for wavelength $\lambda>\lambda_{0}$, with $\lambda_{0}$ and $\beta$ (as well as the dust

${ }^{4}$ Our SED fitting software, SDF, is available at https://github.com/drgmk/sdf. temperature and luminosity) as free parameters. Physically, this extra factor accounts for the fact that the disc emission originates from a distribution of dust grain sizes, with grains emitting less efficiently at wavelengths larger than their own size. We model a single system, HD 95698, with two separate dust components (see e.g. Kennedy \& Wyatt 2014), as this gives a significantly better fit to its Spitzer Infrared Spectrograph (IRS; Houck et al. 2004) spectrum.

Our approach differs slightly from the most common method of SED fitting, which is to choose some cut-off wavelength $(\sim 10 \mu \mathrm{m})$, first using all photometry at shorter wavelengths to fit the stellar component, then extrapolate the resulting stellar model to farinfrared wavelengths. The choice of cut-off wavelength could, however, affect the stellar model, for example, if warm dust were present in a system, resulting in excess mid-infrared flux. Fitting the stellar and dust components simultaneously allows us to obtain a far-infrared photospheric flux prediction without the need to choose a cut-off wavelength. Most of the 'dust' components will in fact be insignificant - that is, consistent with zero emission, and barely contributing to the total flux. However, for systems whose excesses we conclude are significant, the dust model can then be used to characterize the inferred debris disc.

Thus, it must next be decided whether each SED should be considered to show a significant excess of infrared emission. We choose to focus only on excesses at 70 and $100 \mu \mathrm{m}$, corresponding to typical dust temperatures of order $30-200 \mathrm{~K}$. It is possible for systems to host warmer dust, which would be better searched for at mid-infrared wavelengths. However, such warm dust cannot generally be a direct tracer of planetesimal belts in the same way as the cooler dust that we are searching for. This is because a belt of planetesimals at the inferred distance of the warm dust should generally have collisionally depleted to below observable levels on a short time-scale (e.g. Wyatt 2008). It has been suggested that warm dust could either have originated from planetesimals scattered inwards from a more distant belt (Wyatt et al. 2007) or in situ from collisions between planetary embryos (Rhee, Song \& Zuckerman 2008). Systems with excesses in the mid-infrared only are rare (e.g. HD 69830: Lisse et al. 2007; Marshall et al. 2014). Note that BD +20307 , which is well known to host warm dust (Song et al. 2005), is in our sample, though this system also has an excess of far-infrared emission. The key point is that warm dust and cool dust can be regarded as distinct phenomena, and so for 

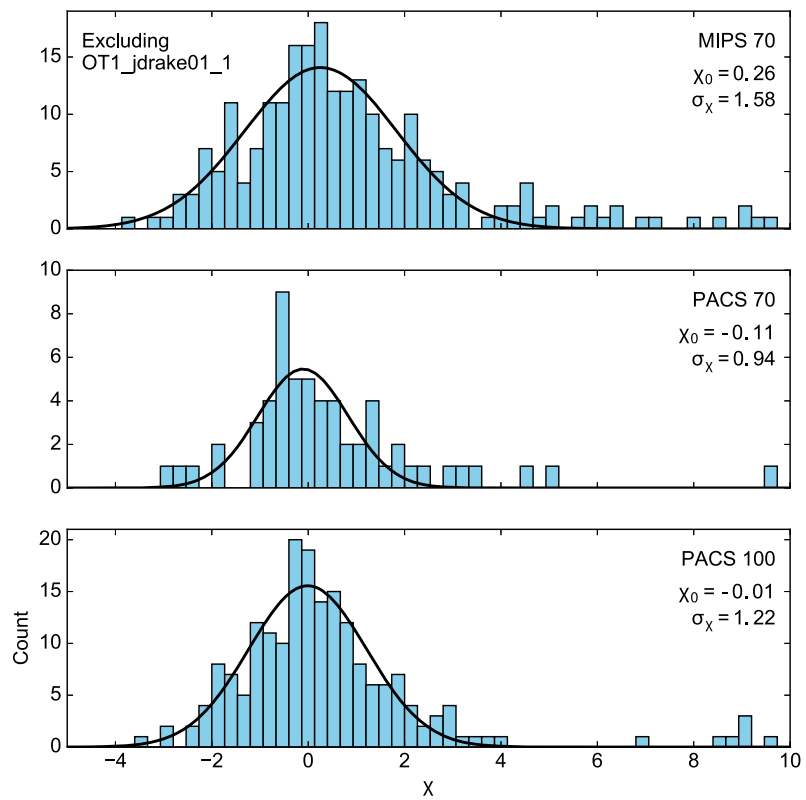

Figure 2. Distributions of photometric significance $\chi$ (bars) for all systems in our sample other than those from the OT1_jdrake01_1 survey, with best-fitting Gaussians (curves) overplotted. Values of $\chi$ greater than 10 are not shown. The upper, middle, and lower panels show photometry from the MIPS $70 \mu \mathrm{m}$, PACS $70 \mu \mathrm{m}$, and PACS $100 \mu \mathrm{m}$ bands, respectively. The annotations display the means $\chi_{0}$ and standard deviations $\sigma_{\chi}$ of the Gaussian fits.

consistency and ease of interpretation we restrict our attention to the latter. While we only search for excesses at 70 and $100 \mu \mathrm{m}$, shorter wavelength photometry - for example, from the Wide-field Infrared Survey Explorer (WISE; Wright et al. 2010) $22 \mu \mathrm{m}$ and MIPS $24 \mu \mathrm{m}$ bands - is useful for constraining dust temperatures where an excess is detected.

A useful measure of the significance of an excess at a given wavelength $\lambda$ is the quantity $\chi$, defined as

$\chi=\frac{F_{v, \mathrm{obs}}-F_{\nu, \mathrm{pred}}}{\sqrt{\sigma_{\mathrm{obs}}^{2}+\sigma_{\mathrm{pred}}^{2}}}$,

where $F_{v, \text { obs }}$ is the observed flux density at wavelength $\lambda, F_{v \text {,pred }}$ is the flux density expected based on the best-fitting stellar model alone, and $\sigma_{\text {obs }}$ and $\sigma_{\text {pred }}$ are the associated uncertainties. The values of $F_{v \text {,pred }}$ and $\chi$ in the three far-infrared bands we are focusing on are shown in Table 2, along with the measured photometry. For almost all systems, the $\sigma_{\text {pred }}$ values (which are not shown)

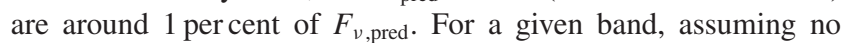
systematic offsets and accurately estimated uncertainties, the $\chi$ values for an unbiased set of observations would be expected to follow a near Gaussian distribution with mean $\chi_{0}=0$ and standard deviation $\sigma_{\chi}=1$, with any observations with $\chi>3$ considered to represent significant excesses. A relatively small proportion of stars host detectable levels of debris - around 20 per cent for single stars, as discussed in Section 1 - hence the approximately Gaussian shape. In practice, we will use the observed $\chi$ distributions to define our excess criterion, as doing so automatically accounts for underestimated or overestimated uncertainties and systematic effects - issues which may not be apparent when fitting individual SEDs.

In Fig. 2, we show $\chi$ distributions for the three bands in which we will search for excesses, along with the best-fitting Gaussians

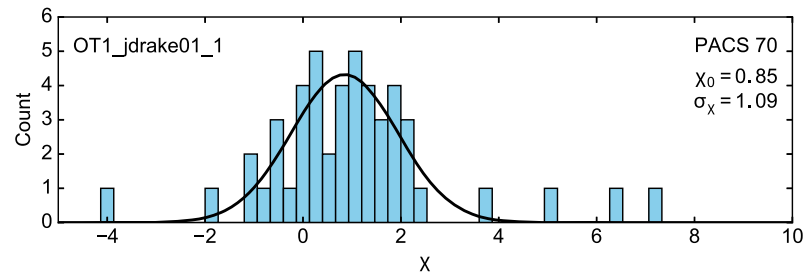

Figure 3. Same as Fig. 2, but only for systems from the OT1_j drake01_1 survey. Only PACS $70 \mu \mathrm{m}$ photometry is shown, as only two of these systems have MIPS $70 \mu \mathrm{m}$ observations and none have PACS $100 \mu \mathrm{m}$ observations.

calculated using the least-squares method. Note that this figure excludes systems from the OT1_jdrake01_1 survey; we find that the significances for those systems are distributed quite differently to the rest of the sample, and will therefore treat them separately (see next). Fig. 2 shows that for MIPS $70 \mu \mathrm{m}$, and to a lesser extent PACS $100 \mu \mathrm{m}$, the $\chi$ distributions for the bulk of the sample are somewhat broader than expected, with $\sigma_{\chi}>1$. This suggests that the uncertainties on either the predicted or observed fluxes in those bands have been underestimated. It could be the case that our estimates of the repeatability of the observations, particularly for MIPS $70 \mu \mathrm{m}$, are simply too optimistic. Some other possible explanations for the broadness of the distributions lie in the fact that our sample is comprised of binaries: First, the fact that we model each system with only a single stellar component could introduce extra uncertainty on the model fluxes. Secondly, some systems will have angular separations such that they are resolved into individual components at optical wavelengths, but not in the far-infrared by MIPS and PACS due to the relatively large beam sizes of these instruments. Thus, some subset of systems will appear to have higher far-infrared significances than they really do - an effect that will tend to both broaden and positively offset the $\chi$ distributions. At 18 arcsec, the MIPS $70 \mu \mathrm{m}$ beam size is the largest of the three far-infrared bands, so this resolution effect should have the greatest impact on the MIPS $70 \mu \mathrm{m}$ significance distribution, consistent with Fig. 2.

In Fig. 3, we plot the PACS $70 \mu \mathrm{m} \chi$ distribution for the OT1_jdrake01_1 systems alone; there are insufficient data in the other two bands to make useful histograms for these systems. This distribution has a large positive offset $\chi_{0}=0.85$, which is not present in the rest of the sample (cf. Fig. 2). Matranga et al. (2010) observed that close binaries often have warm infrared excesses, which are not due to debris discs in the typical sense, and the offset we observe in the OT1_jdrake01_1 PACS data could be related to that phenomenon (see Section 5.3).

To account for the different spreads and offsets of the distributions, for the bulk of the sample, we require a system to have $\chi>\chi_{0}+3 \sigma_{\chi}$ in at least one of MIPS $70 \mu \mathrm{m}$, PACS $70 \mu \mathrm{m}$, and PACS $100 \mu \mathrm{m}$ for its excess to be considered significant. We additionally require that $\chi>3$, since if this is not satisfied then the dust temperature of the model will be poorly constrained by the SED fitting. This second criterion is only relevant to PACS $70 \mu \mathrm{m}$, as the other two bands have $\chi_{0}+3 \sigma_{\chi}>3$. In practice, requiring $\chi>3$ only eliminates a single system that would otherwise be considered to host a disc: HD 81809, which has $\chi=2.90$ in PACS $70 \mu \mathrm{m}$. For the OT1_jdrake01_1 systems, we search only for PACS $70 \mu \mathrm{m}$ excesses, using the same criteria but different distribution parameters as shown in Fig. 3. Note that we treat the bands independently, without giving special treatment to systems with excesses in multiple bands. For example, while it could be argued that a system with significances below but close to the 
threshold in two different bands shows sufficient evidence for a disc, we would not classify such a system as disc bearing using our criteria. As different systems in our sample have observations in different bands, taking such cases into account consistently would require defining different excess criteria for each possible combination of observations in the three far-infrared bands.

There is one system in the sample to which we give special consideration in deciding whether a disc is present. HD 19356 (Algol) has a significant MIPS $70 \mu \mathrm{m}$ excess with $\chi=5.12$, which could be linked to the system's variable millimetre emission rather than originating from a circumstellar disc (Holland et al. 2017). Though there is no direct evidence against the presence of dust in the system, we take a conservative approach and, for the purpose of this paper, do not consider this system to host a debris disc.

In the following section, we present the results on the occurrence and properties of debris discs in our sample that follow from the SED analysis described above.

\section{RESULTS}

Using the methods outlined in the previous section, we are able to come to a conclusion about which systems in our sample are seen to host Kuiper belt-like debris discs, and to quantify the temperatures and luminosities of those discs. In this section, we first present these inferred disc properties and consider their implications for dynamical stability, then perform a statistical analysis to investigate whether the stellar orbits affect the detectability of debris in a significant way.

\subsection{Disc properties}

We find that 38 systems in our sample host debris discs, which are characterized by their fractional luminosity $f$ (i.e. the ratio of their luminosity to the stellar luminosity) and their temperature $T_{\text {disc }}$. These parameters are listed in Table 3 and plotted in Fig. 4. The uncertainties shown in this figure are calculated as half the difference between the 84th and 16th percentiles of the posterior distributions.

Table 3. Results of SED fitting for the 38 systems we find to have a significant infrared excess. The table shows the name of each system, its stellar luminosity, and the disc parameters $T_{\mathrm{disc}}, f, r_{\mathrm{bb}}$, and $r_{\mathrm{disc}}$ as defined in the text of Section 4 with their associated uncertainties. HD 95698 is listed twice because we model it with two dust components of different temperatures. A machine-readable version of this table is available online.

\begin{tabular}{|c|c|c|c|c|c|c|c|c|c|}
\hline Name & $L / L_{\odot}$ & $T_{\mathrm{disc}} / \mathrm{K}$ & $\Delta T_{\text {disc }} / \mathrm{K}$ & $f$ & $\Delta f$ & $r_{\mathrm{bb}} / \mathrm{au}$ & $\Delta r_{\mathrm{bb}} / \mathrm{au}$ & $r_{\text {disc }} / \mathrm{au}$ & $\Delta r_{\text {disc }} / \mathrm{au}$ \\
\hline HD 1404 & 23.1 & 142 & 4 & $1.3 \mathrm{E}-05$ & $9.2 \mathrm{E}-07$ & 19 & 1 & 34 & 2 \\
\hline HD 8997 & 0.5 & 46 & 8 & $2.8 \mathrm{E}-05$ & 3.7E-06 & 26 & 10 & 177 & 72 \\
\hline HD 11171 & 5.8 & 73 & 13 & $6.3 \mathrm{E}-06$ & $1.7 \mathrm{E}-06$ & 36 & 14 & 105 & 42 \\
\hline HD 13161 & 72.8 & 86 & 2 & 2.7E-05 & 6.7E-07 & 89 & 4 & 108 & 5 \\
\hline HD 14082B & 1.1 & 105 & 2 & $2.4 \mathrm{E}-04$ & $8.0 \mathrm{E}-06$ & 7.3 & 0.2 & 39 & 1 \\
\hline HD 16628 & 37.6 & 97 & 7 & $1.6 \mathrm{E}-05$ & $1.4 \mathrm{E}-06$ & 50 & 7 & 77 & 10 \\
\hline HD 17094 & 10.8 & 157 & 56 & $5.8 \mathrm{E}-06$ & $2.8 \mathrm{E}-06$ & 10 & 8 & 23 & 19 \\
\hline HD 17925 & 0.4 & 99 & 6 & 4.9E-05 & $5.7 \mathrm{E}-06$ & 5 & 1 & 37 & 5 \\
\hline HD 19994 & 4.1 & 34 & 8 & 4.2E-06 & 4.4E-07 & 146 & 66 & 484 & 220 \\
\hline HD 20320 & 11.7 & 70 & 2 & $1.8 \mathrm{E}-05$ & $8.9 \mathrm{E}-07$ & 54 & 3 & 124 & 8 \\
\hline HD 20807 & 1.0 & 198 & 36 & $1.1 \mathrm{E}-05$ & $3.5 \mathrm{E}-06$ & 2 & 1 & 10 & 3 \\
\hline HD 21242 & 7.9 & 527 & 126 & $6.2 \mathrm{E}-04$ & $4.5 \mathrm{E}-04$ & 0.8 & 0.4 & 2 & 1 \\
\hline HD 33262 & 1.5 & 132 & 35 & $1.8 \mathrm{E}-05$ & $3.3 \mathrm{E}-06$ & 6 & 4 & 27 & 20 \\
\hline HD 35850 & 1.8 & 78 & 3 & $4.2 \mathrm{E}-05$ & $2.6 \mathrm{E}-06$ & 17 & 1 & 75 & 6 \\
\hline HD 95698 & 8.6 & 211 & 31 & $2.5 \mathrm{E}-05$ & 3.8E-06 & 5 & 1 & 13 & 3 \\
\hline HD 95698 & 8.6 & 47 & 3 & 8.7E-05 & $3.9 \mathrm{E}-06$ & 103 & 10 & 262 & 25 \\
\hline HD 102647 & 13.6 & 118 & 1 & $2.2 \mathrm{E}-05$ & $4.6 \mathrm{E}-07$ & 21 & 1 & 45 & 1 \\
\hline HD 113337 & 4.1 & 55 & 1 & $9.1 \mathrm{E}-05$ & 2.7E-06 & 53 & 1 & 174 & 4 \\
\hline HD 118216 & 21.0 & 211 & 16 & $1.8 \mathrm{E}-05$ & $1.7 \mathrm{E}-06$ & 8 & 1 & 15 & 2 \\
\hline HD 119124 & 1.6 & 56 & 2 & $4.5 \mathrm{E}-05$ & 4.2E-06 & 32 & 2 & 146 & 11 \\
\hline HD 127762 & 33.4 & 85 & 4 & $9.7 \mathrm{E}-06$ & $5.8 \mathrm{E}-07$ & 62 & 6 & 99 & 10 \\
\hline HD 131511 & 0.5 & 47 & 12 & $3.8 \mathrm{E}-06$ & 8.9E-07 & 26 & 14 & 175 & 95 \\
\hline HD 139006 & 71.4 & 124 & 2 & $1.3 \mathrm{E}-05$ & $4.6 \mathrm{E}-07$ & 42 & 2 & 52 & 2 \\
\hline HD 150682 & 6.5 & 34 & 4 & $1.6 \mathrm{E}-05$ & $2.3 \mathrm{E}-06$ & 167 & 47 & 470 & 132 \\
\hline HD 165908 & 2.9 & 45 & 4 & $1.6 \mathrm{E}-05$ & $9.2 \mathrm{E}-07$ & 64 & 11 & 241 & 40 \\
\hline HD 172555 & 8.0 & 200 & 1 & $3.8 \mathrm{E}-04$ & 2.7E-06 & 5.5 & 0.1 & 14.4 & 0.2 \\
\hline HD 181296 & 21.6 & 134 & 2 & $2.1 \mathrm{E}-04$ & 7.6E-06 & 20 & 1 & 37 & 1 \\
\hline HD 196544 & 22.7 & 107 & 2 & $2.8 \mathrm{E}-05$ & 7.2E-07 & 33 & 1 & 59 & 2 \\
\hline HD 196885 & 2.7 & 24 & 7 & $2.8 \mathrm{E}-06$ & 7.1E-07 & 226 & 135 & 868 & 517 \\
\hline HD 202444 & 10.6 & 294 & 105 & $1.8 \mathrm{E}-05$ & $1.8 \mathrm{E}-05$ & 3 & 2 & 7 & 5 \\
\hline HD 206860 & 1.2 & 90 & 7 & $8.5 \mathrm{E}-06$ & $6.3 \mathrm{E}-07$ & 11 & 1 & 55 & 7 \\
\hline HD 207129 & 1.2 & 45 & 1 & $9.1 \mathrm{E}-05$ & $3.2 \mathrm{E}-06$ & 43 & 1 & 217 & 6 \\
\hline HD 213398 & 37.2 & 122 & 21 & $1.4 \mathrm{E}-05$ & $1.3 \mathrm{E}-06$ & 31 & 9 & 47 & 14 \\
\hline HD 213845 & 3.5 & 75 & 17 & $3.5 \mathrm{E}-06$ & $4.5 \mathrm{E}-07$ & 24 & 12 & 84 & 41 \\
\hline HD 216956 & 16.6 & 69 & 3 & 7.6E-05 & 4.3E-06 & 66 & 7 & 134 & 14 \\
\hline HD 223352 & 27.5 & 173 & 3 & 2.3E-05 & 7.3E-07 & 13.6 & 0.5 & 23 & 1 \\
\hline HIP 8920 & 3.2 & 279 & 3 & $3.0 \mathrm{E}-02$ & 7.8E-04 & 1.78 & 0.04 & 6.4 & 0.1 \\
\hline HIP 11437 & 0.2 & 71 & 1 & $1.2 \mathrm{E}-03$ & 4.0E-05 & 7.5 & 0.2 & 68 & 2 \\
\hline HIP 17962 & 0.4 & 69 & 5 & $1.4 \mathrm{E}-04$ & $2.6 \mathrm{E}-05$ & 10 & 1 & 75 & 11 \\
\hline
\end{tabular}




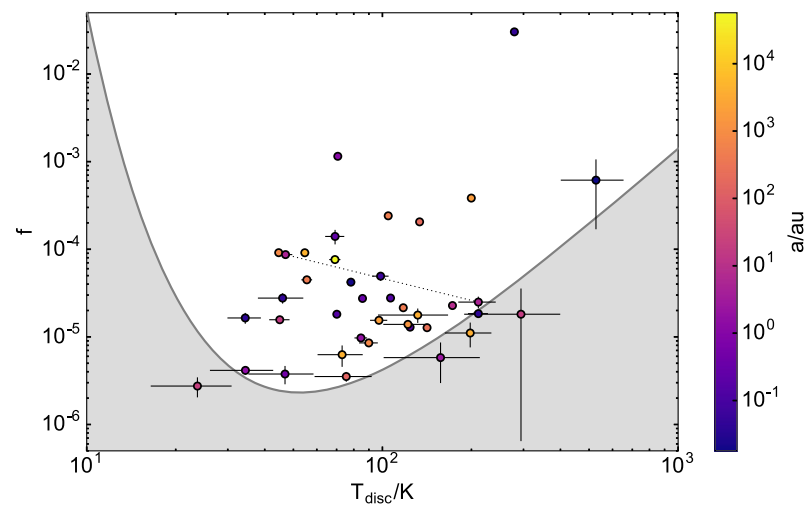

Figure 4. Fractional luminosity versus disc temperature for our full sample. The points are coloured by stellar separation; we use the smallest separation in the system for triple and higher order systems. We model HD 95698 as a two-temperature disc, and the two components are joined with a dotted line. The grey region shows the part of parameter space in which a disc around a typical star in our sample would be undetectable (see Section 4.1 for details); systems lying close to the boundary of this region have comparatively large uncertainties.

The grey shading shows the region of parameter space in which we would not expect a disc around a typical star in our sample to be detectable by PACS at $70 \mu \mathrm{m}$. To estimate the detection threshold, we used equation (8) of Wyatt (2008), taking the sensitivity to be $5 \mathrm{mJy},{ }^{5}$ and the stellar distance and luminosity to be the median values in our sample: $23 \mathrm{pc}$ and $2.1 L_{\odot}$, respectively. Thus, it is not surprising that several systems lie slightly below this threshold since the detection threshold for some stars will be lower than that of a median star. It is none the less a useful guideline, explaining the general form of the region where our discs lie, and why discs in certain parts of parameter space (i.e. near the threshold, where the detections have a lower signal-to-noise ratio) have comparatively large uncertainties. The points in Fig. 4 are coloured by stellar separation $a$, and from this it does not appear that $f$ or $T_{\text {disc }}$ are strongly influenced by $a$ in the region where discs are detected.

\subsection{Dynamical stability}

Next, we wish to assess the dynamical stability of the discs. To do so, we need to establish where in the system they lie, i.e. their radii. We first convert the temperatures obtained from SED modelling into blackbody disc radii $r_{\mathrm{bb}}$ by assuming that the dust acts as a perfect absorber and emitter in thermal equilibrium with the stellar radiation (e.g. Wyatt 2008). Radii calculated in this way, however, will underestimate the true radii of the discs by an amount that depends on the stellar luminosity $L$. The smallest grains in a disc are inefficient emitters, and so the more small grains that are present, the greater the departure from blackbody behaviour. The factor by which the blackbody radius underestimates the true radius is thus greater for lower luminosity stars because brighter stars can remove larger grains via their radiation pressure (e.g. Burns, Lamy \& Soter 1979). For Sun-like stars, this correction factor can be as large as $\sim 5$ (Pawellek \& Krivov 2015), which could make the difference between dust appearing to lie in a stable and an unstable region, and is thus important to account for. To estimate the true disc radii $r_{\text {disc }}$, we use the prescription of Pawellek \& Krivov (2015) - specifically,

\footnotetext{
${ }^{5}$ See table 3.2 of the PACS Observer's Manual, http://herschel.esac.esa.int/ Docs/PACS/html/pacs_om.html.
}
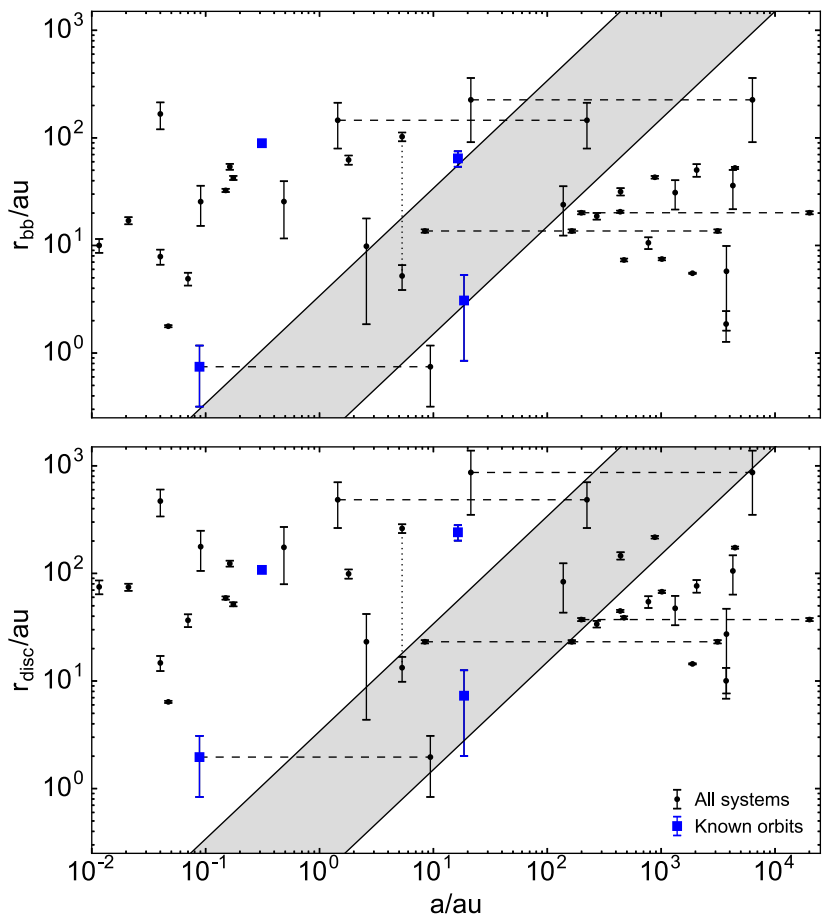

Figure 5. Disc radius versus stellar separation for our full sample. The upper panel shows blackbody disc radii, while the radii in the lower panel have been corrected for inefficient grain emission according to Pawellek \& Krivov (2015). Systems with more than two components, and hence more than one separation, are connected by the dashed lines. We model HD 95698 as a two-temperature disc, and the two components are joined with a dotted line. HD 216956 (Fomalhaut) is the most widely separated system in our sample, and lies beyond the range of separations shown. Systems with highly graded VB6 orbits are plotted as the large square points. The grey shading marks the approximate region of dynamical instability for planetesimals, assuming an equal-mass binary with an eccentricity of 0.4 , as in Rodriguez et al. (2015). Around 24 per cent of the discs we find appear to be unstable based on the lower panel.

we multiply our blackbody radii $r_{\mathrm{bb}}$ by the factor $\Gamma$ defined in their equation (8), assuming that the dust is composed of a mixture of astrosilicate and ice. Note that in addition to the uncertainty arising from the need to assume a particular dust composition, there is some inherent scatter in the relation between $\Gamma$ and $L$, and so while we do on average expect the correction to improve our estimates of the disc radii there may be individual systems for which the blackbody radius is closer to the true value.

Both $r_{\mathrm{bb}}$ and $r_{\text {disc }}$ are listed in Table 3 and plotted as functions of stellar separation in Fig. 5. Also shown in this figure, with grey shading, is the approximate dynamically unstable region of parameter space as calculated by Rodriguez et al. (2015), who used the results of Holman \& Wiegert (1999) and assumed equal stellar masses and an orbital eccentricity of 0.4 . The boundaries of this region are at 0.15 and 3.4 times the stellar separation. Note that a system lying in this region can only be considered definitively unstable if the value of $a$ used is the true semimajor axis of the stellar orbit. As discussed in Section 2, this is not in fact the case for most systems in our sample. For visual binaries - i.e. those towards the right-hand side of Fig. 5 - generally only the projected separation is known, and this is used for $a$. In contrast, the $a$ values for spectroscopic binaries, which lie towards the left-hand side, will have been derived from known periods. While the conversion from 
period to semimajor axis does require an estimate of the total stellar mass $M$, this can be established from stellar spectra sufficiently accurately for our purposes (up to a factor $\sim 2$ for equal-mass binaries), and the dependence on $M$ is not strong $\left(a \propto M^{1 / 3}\right)$, which means that semimajor axis estimates for spectroscopic binaries will generally be reliable. Thus, while in all but a few cases $a$ is not a direct measurement of the semimajor axis, systems lying further towards the left-hand side of the plot will generally have an $a$ value closer to their true semimajor axis.

Of the 38 discs we find, 9 have an $r_{\text {disc }}$ placing them in the nominally unstable region: HD 19994, HD 21242, HD 95698, HD 119124, HD 181296, HD 202444, HD 207129, HD 213845, and HD 223352. We generally expect $r_{\text {disc }}$ to give a better estimate of the disc radii, and thus a better indication of stability, than $r_{\mathrm{bb}}$. However, to allow a direct comparison with previous work we note that if the blackbody radii were to be interpreted as true disc radii, then we would conclude that the only unstable systems were HD 19994, HD 95698, HD 202444, HD 213845, and HD 223352. That is, correcting for inefficient grain emission moves four systems up into the unstable region, without moving the other five far enough out to be considered stable.

Assuming binomial uncertainties (e.g. Burgasser et al. 2003), the nine potentially unstable systems constitute $24_{-6}^{+8}$ per cent of the discs. For comparison, of the 71 systems considered to host discs in Trilling et al. (2007), Rodriguez \& Zuckerman (2012), and Rodriguez et al. (2015) combined, a total of 13 were concluded to be unstable, or $18_{-3}^{+5}$ per cent. While our derived apparent instability fraction is therefore consistent with the combined results of these three studies, our specific set of unstable discs is quite different. In fact, in many cases we do not detect a disc in the previously proposed unstable systems at all, which emphasizes the importance of revisiting the conclusions of previous work. For example, none of the three systems with 'unstable discs' from Trilling et al. (2007) - HD 46273, HD 80671, and HD 127726 - in fact satisfy our excess criterion. Note that while an MIPS $70 \mu \mathrm{m}$ observation of HD 127726 exists from Trilling et al. (2007), we do not include the corresponding flux in our SED fitting because it is $4.4 \sigma$ (or a factor of $\sim 3$ ) above the OT2_gkennedy_2 PACS $70 \mu \mathrm{m}$ flux, probably due to contamination from a background source that is clearly visible in the PACS image $\sim 20$ arcsec away; this is likely the reason for our different conclusions for this particular system. Similarly, none of the three 'unstable' systems from Rodriguez \& Zuckerman (2012) that are in our sample (HD 56986, HD 73752, and HD 174429) appear to have a significant excess. Of the four 'unstable' Rodriguez et al. (2015) systems, our results agree that HD 223352 and HD 19994 do seem to host unstable dust, but we conclude that the disc of HD 165908 (99 Her) is in a stable location based on either $r_{\text {disc }}$ or $r_{\mathrm{bb}}$ (see also Kennedy et al. 2012a) and that HD 133640 does not have a significant infrared excess. For reference, the far-infrared significances of the systems previously thought to host an unstable disc that we do not consider to have an excess are displayed in Table 4.

Fig. 5 also highlights (as larger, the square points) those systems for which the stellar orbits can be considered well known, i.e. those listed in VB6 with grade 1 or 2, as discussed in Section 2. These are the systems for which the plotted stellar separations are true semimajor axes, which allows a stronger conclusion to be drawn about their discs' stability than if only the projected separation were known. Recall also that spectroscopic binaries are not subject to projection effects, which in practice means that although they do not have directly measured semimajor axes like the highly graded VB6 systems, the horizontal positions of most binaries with $a$ less
Table 4. Far-infrared significances of the seven systems previously considered to host unstable dust and for which we do not detect an excess. Recall that the excess thresholds are set by Figs 2 and 3, and can thus exceed $\chi=3$. In particular, the MIPS $70 \mu \mathrm{m}$ threshold is $\chi=5.00$.

\begin{tabular}{lccc}
\hline Name & MIPS 70 $\mu \mathrm{m}$ & $\begin{array}{c}\chi \\
\text { PACS 70 } \mu \mathrm{m}\end{array}$ & PACS 100 $\mu \mathrm{m}$ \\
\hline HD 46273 & 2.52 & -0.35 & - \\
HD 56986 & -0.62 & - & 1.06 \\
HD 73752 & 0.15 & - & -0.06 \\
HD 80671 & 1.91 & 1.08 & - \\
HD 127726 & - & -0.52 & - \\
HD 133640 & 3.74 & - & 2.03 \\
HD 174429 & 0.62 & 1.78 & 1.02 \\
\hline
\end{tabular}

than around $1 \mathrm{au}$ in Fig. 5 are well constrained, allowing us to conclude that such systems are very likely stable.

The disc-bearing known VB6 orbit systems are HD 13161 (beta Tri), HD 165908 (99 Her), HD 202444 (tau Cygni), and HD 21242 (UX Ari). The first two of these systems are well known from previous work to host discs (Kennedy et al. 2012a,b), and lie well outside the unstable zone. The third, HD 202444, is a new detection from the OT2_gkennedy_2 survey, and lies in the unstable zone in Fig. 5. Finally, HD 21242, one of the OT1_jdrake01_1 targets, is a triple system, and it is only the AaAb component that has a known orbit. Fig. 5 shows that the dust is sufficiently far out to be stable to perturbations from the AaAb pair, though it does lie just inside the nominally unstable region of the less well-constrained $\mathrm{AB}$ pair.

Rodriguez et al. (2015) noted that two possible effects that could cause stable discs to appear unstable in a plot of $r_{\text {disc }}$ against $a$ are inefficient grain emission (i.e. the blackbody disc radius underestimating its true radius) and orbital projection (i.e. lack of knowledge of the true stellar semimajor axis). We have corrected for the former effect to the extent that this is possible and still found that a significant fraction of discs lie in the unstable region. In addition, while the number of disc-bearing binaries whose semimajor axes are well known is very limited, we found that one out of the four such discs in our sample appears unstable.

\subsection{Assessment of potentially unstable systems}

In the previous subsection, we identified nine systems that, based on their values of $r_{\text {disc }}$, appear to host unstable dust. As these systems are of particular interest, here we discuss each of them individually and assess the evidence that their discs truly are unstable.

HD 19994 - this is a triple star system; the B and C components are on a close orbit ( $a \sim 1.5 \mathrm{au}$, from VB6), while the BC pair is at a projected separation of $a \sim 220$ au from the A component (Rodriguez et al. 2015). We derive a disc radius of $480 \pm 220$ au. The simplified picture presented by Fig. 5 suggests that the disc is stable to perturbations from the $\mathrm{BC}$ pair but formally lies just inside the unstable region of the A-BC system. Wiegert, Faramaz \& Cruz-Saenz de Miera (2016) studied HD 19994 in detail, and using radiative transfer modelling and $\mathrm{N}$-body simulations of different possible disc geometries, they concluded that the disc, if real, is likely circumsecondary, i.e. orbiting the BC pair, and in a stable location. They also noted that there is a 2.3 per cent probability that the $100 \mu \mathrm{m}$ excess is due to contamination from a background galaxy. This contamination probability depends on wavelength and PSF size, and so its exact value will vary within our sample depending on the data that exist for each star. However, given our sample size of several hundred systems, assuming a value on the 


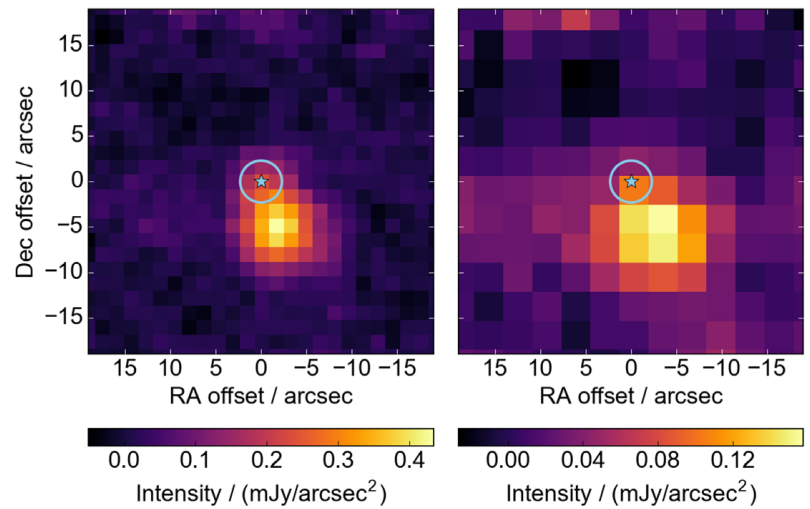

Figure 6. Cut-outs of the PACS images of HD 19994, at $100 \mu \mathrm{m}$ (left) and $160 \mu \mathrm{m}$ (right). The expected stellar position at the time of observation is marked, along with an error circle reflecting the Herschel pointing uncertainty of $2.3 \mathrm{arcsec}$. The $100 \mu \mathrm{m}$ image appears to show two sources: one at the expected stellar position, and another, brighter source around 5 arcsec away. In the $160 \mu \mathrm{m}$ image, only the brighter of the two sources is visible. This may suggest that the image is contaminated by a background galaxy.

order of 1 per cent it is expected that several stars in our sample will be affected by background galaxies. For this system in particular, the PACS images, shown in Fig. 6, do hint at background contamination: at $100 \mu \mathrm{m}$ there appears to be one source very close to the expected stellar position at the time of observation, with another, brighter source around 5 arcsec away. At $160 \mu \mathrm{m}$, only the latter of these appears to be detected. Note that in Fig. 6, the stellar position shown is calculated using Hipparcos proper motions; the error circle around the star has radius $2.3 \mathrm{arcsec}$, reflecting the Herschel pointing uncertainty. ${ }^{6}$ PSF fitting favours subtracting the brighter source, but it is possible that this is in fact a galaxy, while the less bright source is the star. In summary, we do not consider the evidence that this system genuinely contains unstable dust, or perhaps a disc at all, to be compelling.

HD 21242 (UX Ari) - another triple system, with AaAb on a close orbit and a more distant $\mathrm{B}$ component. Estimating the $\mathrm{AaAb}$ and $\mathrm{AB}$ semimajor axes from SB9 periods as outlined in Section 2 gives 0.08 and 9 au, respectively. The periods we used are those calculated by Duemmler \& Aarum (2001) using radial velocity observations; the $\mathrm{AB}$ period is based on a fit to the velocity of the centre of mass of the AaAb pair, which they found to be changing systematically with time. The AaAb orbit is listed with grade 2 in VB6, giving a semimajor axis of $0.09 \mathrm{au}$, very close to the value we inferred from the period. $\mathrm{AB}$ also appears in VB6 with a semimajor axis of $33 \mathrm{au}$; as this is graded 5, we take the $\mathrm{AB}$ semimajor axis to be $9 \mathrm{au}$. Note that the $\mathrm{AB}$ component lies very close to the edge of the nominally unstable region in Fig. 5, and using the VB6 value would move the system well into the stable region. We find a disc at $2 \pm 1 \mathrm{au}$. It is the hottest disc in our sample by some margin $\left(T_{\text {disc }} \sim 500 \mathrm{~K}\right)$, and the third brightest $\left(f \sim 6 \times 10^{-4}\right)$, making it somewhat exceptional. Matranga et al. (2010) similarly identified this system as hosting large amounts of very warm dust, and suggested planetary collisions as a potential origin of this dust. We discuss this idea further in Section 5.3. If this system hosts dust that is truly residing in the unstable zone, then binary perturbations

${ }^{6}$ See table 2.1 of the Herschel Observer's Manual, http://herschel.esac.esa. int/Docs/Herschel/html/Observatory.html. from the $\mathrm{AB}$ pair could provide an explanation for the destabilisation of the planets proposed to be responsible. Alternatively, note that the dust is consistent with lying at the boundary of instability (which is formally at $1.4 \mathrm{au}$ ); this could imply that the disc is being truncated at its outer edge by perturbations from the B component.

HD 95698 - a binary with projected separation 5 au (Trilling et al. 2007). As noted in Section 3, inspection of its IRS spectrum motivated us to model this system with two-temperature components. This suggests that the disc likely has significant radial extension and/or multiple belts, though two-temperature discs can also arise from a single narrow belt, in which different grain sizes have different temperatures (Kennedy \& Wyatt 2014). While the cooler component lies well into the stable zone (at $262 \pm 25 \mathrm{au}$ ), the warmer component is formally unstable with $r_{\text {disc }}=13 \pm 3$ au. Given that the boundary of the grey region in Fig. 5 is blurred by the assumption of a fixed stellar mass ratio and eccentricity, and that the warmer component here lies very close to this boundary (which is at $18 \mathrm{au}$ ), the implication may be that the dust responsible for the warm emission is being truncated at its inner edge by binary perturbations. This disc is also resolved at $70 \mu \mathrm{m}$ in the OT2_gkennedy_2 observations, and the details of our image modelling are presented in appendix A. Our conclusions from this modelling are similar to our findings from SED fitting: the image is consistent with a broad disc extending from $101_{-38}^{+72}$ to $318_{-54}^{+43}$ au, corresponding to the cooler component of our SED model. We also allow for a component of unresolved flux, and find that the best-fitting model favours such a component being present at $3 \mathrm{mJy}$ (in addition to the $\sim 5 \mathrm{mJy}$ from the star), around 2.6 per cent as bright as the resolved component; the warmer component of our SED model is likely responsible for this emission. In our SED fit, the warmer component similarly has 2.7 percent of the flux of the cooler component at $70 \mu \mathrm{m}$. As the warmer component is not resolved, the image cannot give a better constraint on its radius than SED modelling, and our conclusion about its stability is not affected.

HD 119124 - another binary, with projected separation $a \sim 440$ au (Rodriguez \& Zuckerman 2012). The excess is unambiguous, with a $14 \sigma$ detection in MIPS $70 \mu \mathrm{m}$. It has not been previously identified as unstable, and if we had taken the disc radius to be equal to the blackbody radius of $32 \pm 2$ au then we would also have concluded that it is stable. As a late F-type star, its stellar luminosity correction factor is large (Pawellek \& Krivov 2015), placing its estimated 'true' disc radius firmly in the unstable region, with $r_{\text {disc }}=145 \pm 11$ au. However, note that the quoted uncertainty of 11 au does not acknowledge the uncertainty inherent in the correction factor, so the disc could be stable if the dust composition is different to that assumed.

HD 181296 (eta Tel) - a triple system, with the secondary companion 200 au away (Rodriguez \& Zuckerman 2012) in projected separation, and a much more distant tertiary companion at $\sim 2 \times 10^{4}$ au (Tokovinin 2018), sufficiently distant that it will not affect the stability of the circumprimary disc. The tertiary companion is HD 181327, which hosts its own debris disc (Lebreton et al. 2012), but as we are focusing on observations of primary stars, we plot both components at our calculated circumprimary disc radius in Fig. 5. We find $r_{\mathrm{bb}}=20 \pm 1$ au and $r_{\text {disc }}=37 \pm 1$ au. Midinfrared images of HD 181296 have revealed a Solar-system-like architecture, with a resolved disc at $24 \pm 8$ au and an unresolved component at $\sim 4$ au (Smith et al. 2009); thus, in this case, the blackbody radius actually provides a slightly better estimate of the true radius. Based on the resolved radius, the disc is not unstable. Note also that the primary is an early A-type star, while the secondary companion is in fact a brown dwarf (Neuhäuser et al. 
2011), meaning that the mass ratio here is particularly extreme. The grey region in Fig. 5 assumes equal masses for both stars, so the unstable region for this particular system will be narrower than the figure suggests.

HD 202444 - this binary system has a semimajor axis of $a=18.6 \mathrm{au}$, from VB6. This is the only potentially unstable system whose orbit is well known. The PACS $70 \mu \mathrm{m}$ data from OT2_gkennedy_2 show an excess with significance $\chi=4.54$. As this is a single-band excess whose signal-to-noise ratio is not particularly high, the disc radius is not well constrained, with $r_{\text {disc }}=7 \pm 5 \mathrm{au}$. Given that the excess is detected by PACS at $70 \mu \mathrm{m}$ but not $160 \mu \mathrm{m}$, by considering the detection limits as a function of disc radius in these two bands we can deduce an upper limit on $r_{\mathrm{bb}}$ of $\sim 100 \mathrm{au}$, corresponding to an $r_{\text {disc }}$ of $\sim 240 \mathrm{au}$. Note that this upper limit appears incompatible with the above quoted disc radius and its uncertainty. This is likely a result of the fact that when a model with only a stellar component is fitted to the system's SED, the AKARI (Ishihara et al. 2010) $9 \mu \mathrm{m}$ flux is $\sim 4 \sigma$ below the photosphere, while the MIPS $24 \mu \mathrm{m}$ flux is $\sim 2 \sigma$ above it. Adding a dust component to the model allows the residuals in both of these bands to be made less significant: The stellar component preferentially has a lower flux than in the star-only model, set by the AKARI $9 \mu \mathrm{m}$ photometry, while the MIPS $24 \mu \mathrm{m}$ flux - which is now even further above the photosphere, at $\sim 7 \sigma-$ forces the dust to a relatively high temperature of $\sim 300 \mathrm{~K}$. It could be the case that the $A K A R I$ photometry is subject to underestimated uncertainties and/or a systematic effect, either of which could make the low flux measured for this system unreliable. If so, the MIPS $24 \mu \mathrm{m}$ excess would not be so significant and the dust could be much cooler, as indicated by the upper limit derived only from consideration of the PACS sensitivities. Despite the poorly constrained radius, as orbital projection is not an issue for this system it may be an interesting target for future observations.

HD 207129 - a binary system with projected separation $a \sim 880$ au (Rodriguez et al. 2015). Its ring-like disc has been resolved both in scattered light, with a measured radius of around 160 au (Krist et al. 2010), and by Herschel, with a measured radius of $140 \pm 30 \mathrm{au}$ (Marshall et al. 2011). Our SED modelling gives a blackbody radius of $43 \pm 1 \mathrm{au}$; as a G-type star, its luminositycorrected radius is considerably larger, at $r_{\mathrm{disc}}=217 \pm 6 \mathrm{au}$. Thus, in this case the corrected radius does indeed allow a better assessment of stability, since it overestimates the Herschel-resolved radius by a factor of only 1.6, while the blackbody radius is around 3.3 times too small. The lower boundary of the formally unstable region at a semimajor axis of $880 \mathrm{au}$ is at $\sim 130 \mathrm{au}$, and so based solely on $r_{\text {disc }}$ the dust would be considered unstable. However, the Herschelresolved radius, which directly measures the true disc size, while still formally unstable, is extremely close to - and in fact consistent with - the boundary. It may thus be the case that the disc is not truly unstable, and either the binary semimajor axis is greater than the projected separation or the disc is being truncated at its outer edge by the binary companion.

HD 213845 - a binary with projected separation $a \sim 140$ au (Rodriguez et al. 2015) and $r_{\mathrm{disc}}=80 \pm 40 \mathrm{au}$. It has a marginal excess in PACS $100 \mu \mathrm{m}$, with $\chi=3.88$, hence the relatively poorly constrained radius. Its MIPS $70 \mu \mathrm{m}$ flux is notably high too, but still below our threshold, with $\chi=3.20$. As with HD 119124, the system lies well into the apparently unstable region in Fig. 5, and there is no obvious reason to disbelieve the excess. The PACS $100 \mu \mathrm{m}$ image is not resolved; while the image has a low signal-to-noise ratio, this may suggest that the true disc radius is smaller than $80 \mathrm{au}$. A disc of this size would have an angular diameter of $7.0 \mathrm{arcsec}$ at this system's distance of $22.7 \mathrm{pc}$, somewhat larger than the PACS beam size of $6.65 \operatorname{arcsec} \times 6.87$ arcsec (Poglitsch et al. 2010).

$H D 223352$ - this is a hierarchical quadruple system. The AB separation is $160 \mathrm{au}$, with the $\mathrm{B}$ component itself being a binary of separation $\sim 8 \mathrm{au}$; the $\mathrm{C}$ component (HD 223340) is seen at a projected separation of $\sim 3100$ au from the AB system (Rodriguez et al. 2015). As noted in that paper, the system is unusual in that two of its constituent stars - A and C - are known to host their own debris discs (Phillips 2011). Fig. 5 is made without any knowledge of which star the detected dust orbits, and so we plot all three separations listed above at the same dust radius: $r_{\mathrm{bb}}=13.6 \pm 0.5 \mathrm{au}$ or $r_{\text {disc }}=23 \pm 1$ au. In fact, inspection of Fig. 5 shows that in our study this system only appears unstable if one plots the close $\mathrm{BaBb}$ separation - however, given the knowledge that the dust is not orbiting the B pair, it becomes clear that this separation is not relevant. The dust is stable to perturbations from the $\mathrm{C}$ component, and is formally stable - but at the boundary $(\sim 24 \mathrm{au})-$ with respect to the B component. The blackbody radius for the circumprimary disc derived by Rodriguez et al. (2015) is somewhat larger than our value, at $27 \mathrm{au}$, which led them to the similar conclusion that the dust is at the boundary of stability (though formally unstable). This could be another case in which either the circumprimary disc is being truncated at its outer edge by perturbations from the $\mathrm{B}$ component or the true binary semimajor axis is greater than the projected stellar separation.

We now summarize our findings from the above consideration of individual systems. The main conclusion here is that while some systems do appear based on Fig. 5 to host unstable discs, a more detailed consideration of these systems highlights the fact that this result should not be taken at face value. For one system (HD 19994), we are doubtful about the existence of a disc, due to possible background contamination. For another system (HD 223352), the disc only appears unstable if we consider the $\mathrm{BaBb}$ separation, but from previous work it is in fact known to be circumprimary. There is also a system (HD 181296) whose disc radius from resolved image modelling differs from our radius from SED fitting and indicates that the disc is actually stable.

For two systems (HD 21242 and HD 95698), our calculated radii place them very close to the boundary of stability, as does the resolved radius of another (HD 207129). In such cases, we are particularly hesitant to conclude that the discs are genuinely unstable. This is because the boundary of instability is not as well defined as it appears in Fig. 5 since the mass ratio and eccentricity vary between systems and are not generally well known. Given the proximity of these systems to the nominal boundary, it could be the case that these discs are stable and being truncated by binary perturbations; however, uncertainty on the true positions of systems in Fig. 5 (as discussed in detail next) means that the evidence for the truncation hypothesis is not strong. We note, though, that it is known that discs undergoing truncation could appear unstable: Thébault, Marzari \& Augereau (2010) found using $N$-body simulations that a circumprimary disc being truncated at its outer edge by a binary companion will extend into the dynamically unstable region to some degree, as a result of collisionally produced small grains that are placed on eccentric orbits by radiation pressure. This can alter the SED of a system with such a disc sufficiently to push its inferred radius into the unstable region, even though the parent planetesimals are stable.

The three remaining systems, which lie well into the unstable region in Fig. 5, are HD 119124, HD 213845, and HD 202444. These may be interesting targets for future observations, however, there are still effects that make their true positions in that figure uncertain. 


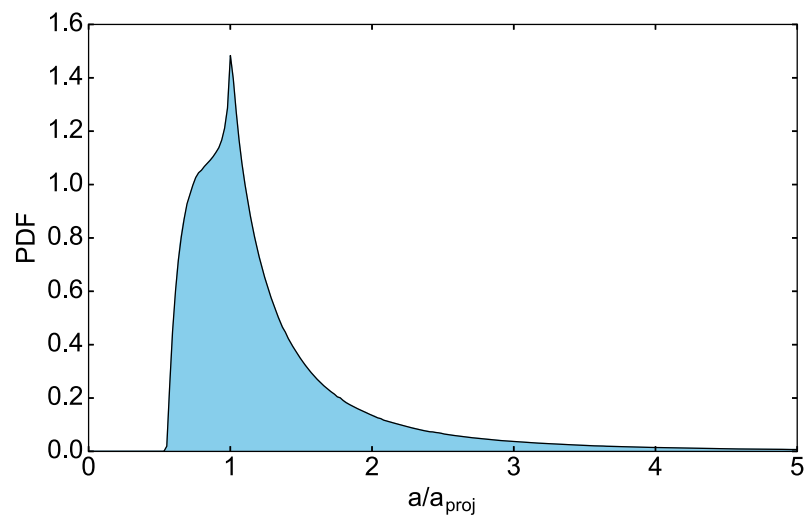

Figure 7. Probability density function (PDF) of the ratio of true semimajor axis to projected separation for a population of binaries with uniformly distributed orbital elements. Eccentrities were taken to be distributed between 0 and 0.8 .

We found by considering the resolved radius of HD 181296 that the 'corrected' disc radius $r_{\text {disc }}$ is not always a better indicator of true disc size than the blackbody radius; this is not surprising given that the correction requires an assumption of dust composition and that the correction factor is known to show some inherent scatter (Pawellek \& Krivov 2015). Thus, the vertical positions of the systems in Fig. 5 are actually less well known than the error bars suggest. We note that the blackbody radii $r_{\mathrm{bb}}$ of HD 213845 and HD 202444 lie at the boundary of instability, and that of HD 119124 lies in the stable region.

There is also the possibility of orbital projection for visual binaries introducing uncertainty on the horizontal positions. To quantify the importance of this effect, we generated $10^{7}$ sets of random uniformly distributed orbital elements, with inclination $I$ ranging from 0 to $\pi / 2$, eccentricity $e$ from 0 to 0.8 , argument of pericentre $\omega$ from 0 to $2 \pi$, and mean anomaly $M$ from 0 to $2 \pi$. The eccentricity distribution we used is an approximation to what has been observed in a real population of binaries (see fig. 14 of Raghavan et al. 2010). We used uniformly distributed mean anomalies rather than true anomalies so that the fact that the stars spend more time near apocentre than near pericentre is accounted for. For each set of elements, we calculated the ratio of the true semimajor axis $a$ to the projected separation $a_{\text {proj }}$, using equation (2.122) of Murray \& Dermott (1998). The resulting distribution of $a / a_{\text {proj }}$ is shown in Fig. 7. It is clear that the distribution is highly skewed, and while the most probable result is that the semimajor axis is equal to the observed projected separation, there is a non-negligible probability that the true value is several times larger. The median value of $a / a_{\text {proj }}$ is $1.06_{-0.29}^{+0.69}$, where the quoted uncertainties are based on the 16th and 84th percentiles of the distribution; its mean is 1.39 . Given the projected separations of HD 119124 and HD 213845, and assuming no uncertainty on their luminosity-corrected disc radii, the distribution in Fig. 7 implies that the probabilities that the true semimajor axes of these systems lie outside the grey region in Fig. 5 are 9.8 per cent and 2.9 per cent, respectively. The actual probabilities of stability will be even larger since, as discussed above, the vertical positions of the points are uncertain.

Given the expected short lifetime of unstable dust, we consider it likely that some combination of projection and uncertainty on the true disc radius is responsible for the apparent instability of HD 119124, HD 213845, and HD 202444. Recall also that Trilling et al. (2007) suggested the inward migration via PR drag of dust produced in a more distant stable planetesimal belt as an origin for unstable

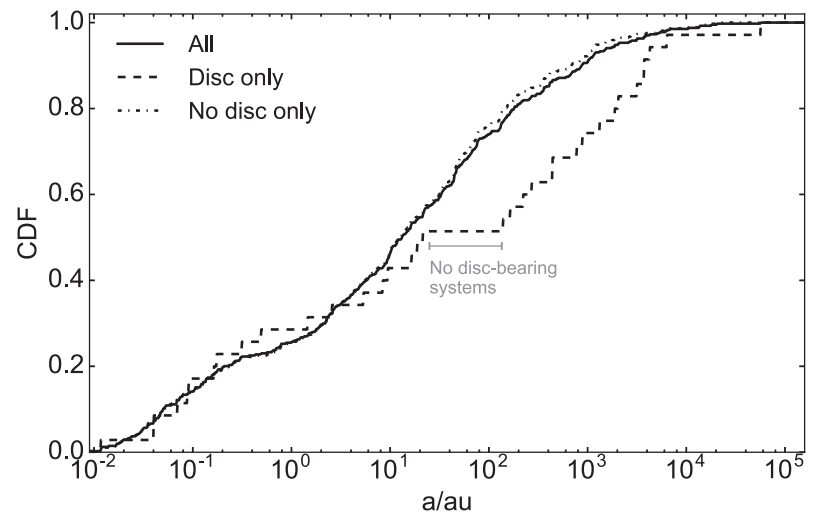

Figure 8. Cumulative distribution functions (CDFs) of stellar separations, for all unbiased systems in our sample, for disc-bearing systems, and for systems without a detectable disc. There is an absence of disc-bearing systems with separations in the range $\sim 25-135 \mathrm{au}$. The grey shading highlights the separation range for which discs are lacking.

dust in binary systems. Aside from the fact that dust is expected to collisionally evolve faster than PR drag can act (as discussed in Section 1), there is the issue that HD 119124, HD 213845, and HD 202444 all have $r_{\text {disc }}<a$ and thus appear to be circumstellar rather than circumbinary, meaning that there are no suitable stable locations for planetesimals from which the dust could drift inwards. We finish by highlighting HD 202444 as perhaps the best candidate for a genuinely unstable system, since it has a known orbit and is thus not subject to horizontal uncertainty, with the caveat that its infrared excess is detected at relatively low signal to noise.

\subsection{Disc statistics}

Having identified and characterized the discs in our sample, we now turn our attention to the issue of whether the likelihood of finding a detectable level of dust in a binary or multiple system depends on its orbit. For the purposes of this subsection, we exclude the 13 systems that we took from Rodriguez \& Zuckerman (2012) and that do not also appear in the samples of Trilling et al. (2007) or Rodriguez et al. (2015). This is because all systems compiled by Rodriguez \& Zuckerman (2012) were included in their study specifically because they were thought from previous work to host debris discs. In contrast, all other sources from which we compile our complete sample are unbiased towards the detection of discs. Thus, including the Rodriguez \& Zuckerman (2012) systems could affect our statistical conclusions, making discs appear to be more common than they really are. Note that although seven of the OT2_gkennedy_2 systems were included in that programme to follow-up on the Trilling et al. (2007) results (see Section 2) - and are therefore part of the OT2_gkennedy_2 sample specifically because they have discs - these systems fall in the unbiased Trilling et al. (2007) sample anyway and are thus not excluded from the statistical analysis. The resulting 'statistical' sample thus contains 328 systems, with a total of 403 stellar separations.

In Fig. 8, we plot the cumulative distribution function (CDF) of stellar separations for the set of all unbiased systems, as well as separate CDFs for the disc-bearing subset and the subset for which no disc is detected. As in Fig. 1, systems of more than two stars are counted multiple times (once at each individual separation). The most striking feature of this plot is that the CDF for disc-bearing systems is constant between around 25 and $135 \mathrm{au}-$ i.e. no discs are detected in systems with separations in that range. The results of 


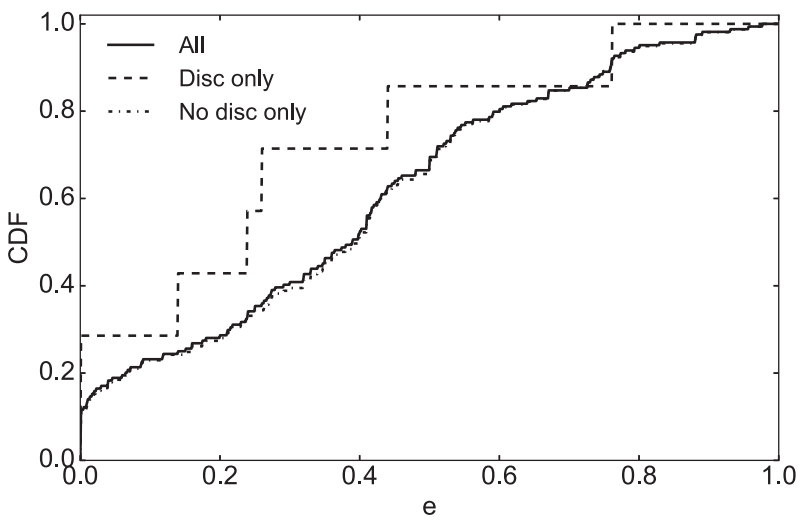

Figure 9. Cumulative distribution functions (CDFs) of orbital eccentricities, for all unbiased systems in our sample with eccentricity information, for disc-bearing systems, and for systems without a detectable disc. The CDFs are statistically indistinguishable.

Rodriguez et al. (2015) similarly hinted that discs are less common in binaries of intermediate separation than in close or wide systems. However, on performing a KS test on the separations of their discbearing and disc-free systems, they obtained a $p$-value of 0.09 , and were thus unable to conclude that the two distributions were in fact different (assuming that a confidence level of 95 percent is required to draw such a conclusion). We performed the equivalent test on our CDFs, yielding a $p$-value of 0.006 . This indicates that the separations of the disc-bearing and disc-free systems are indeed drawn from different distributions, at a confidence level of 99.4 per cent. We therefore conclude that detectable cool debris discs are less common in binary systems with separations between $\sim 25$ and 135 au.

We also performed a KS test on the eccentricities $e$ of the disc-bearing and disc-free systems and found no evidence for a significant difference between their distributions, with a $p$-value of 0.86 if only systems with highly graded VB6 eccentricities are included, or 0.25 for systems with any available eccentricity (either in VB6 with any grade or from the literature). This may suggest that the eccentricity of a binary does not significantly affect its likelihood of hosting detectable dust, but it should also be noted that we only have eccentricity information for 139 of 341 systems. These systems are strongly biased towards having close separations, with 92 per cent of the known eccentricities corresponding to separations of less than $100 \mathrm{au}$. The relatively large $p$-values do not necessarily rule out a dependence of dust detectability on binary eccentricity, but could simply result from the relatively small numbers involved. Eccentricity CDFs for all unbiased systems with eccentricity information, and for disc-bearing and disc-free systems separately, are shown in Fig. 9. These CDFs hint at the result that disc-bearing systems tend to have lower eccentricities than disc-free systems, or equivalently that the occurrence rate of detectable discs is higher for lower eccentricity binaries. If this is a genuine physical effect, it could be a result of the fact that more eccentric binaries will excite orbiting planetesimals to higher eccentricities, thus increasing their relative velocities and reducing their collisional lifetime.

While the general conclusion that debris discs in intermediate separation binaries are somewhat less common is not unexpected based on previous work, we were surprised to find a complete absence of discs over an order of magnitude of separations. Note that Phillips (2011) similarly found an absence of detectable debris discs in binaries with separations of $\sim 3-150$ au using MIPS photometry,

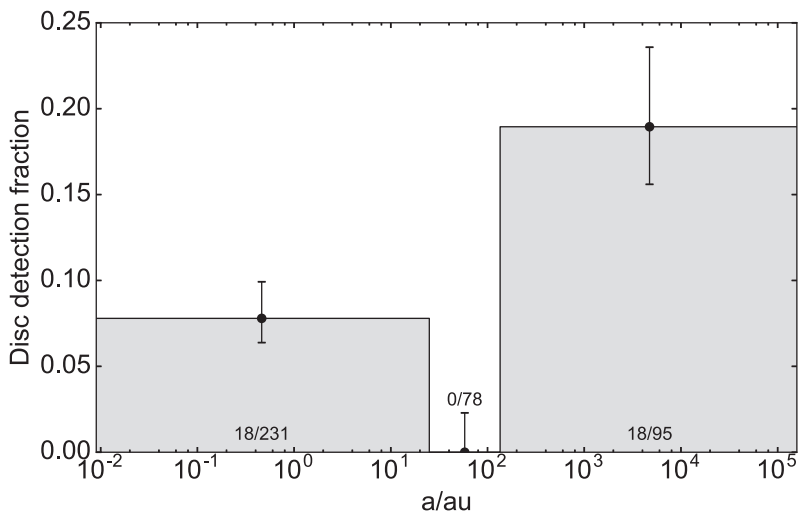

Figure 10. Fraction of unbiased systems in which we detect a debris disc, binned into three stellar separation ranges: $a<25$ au, 25 au $<a<135$ au, and $a>135 \mathrm{au}$. The annotations show the number of disc detections and stellar separations in each bin, with triple and higher order systems counted once per separation.

though for a smaller sample of $\sim 50$ systems, all of which were A-type. As a check on our result, we inspected the SEDs of systems with separations between 25 and $135 \mathrm{au}$, which previous work concluded did have a disc (listed below) individually. From Rodriguez et al. (2015), HD 133640 has both MIPS $70 \mu \mathrm{m}$ and PACS $100 \mu \mathrm{m}$ photometry, neither of which we deem significant; HD 202940 has photometry in these bands as well as PACS $70 \mu \mathrm{m}$, and again none are significantly in excess, though the PACS 100 and $160 \mu \mathrm{m}$ fluxes are both at $3.3 \sigma$. Infrared excesses peaking beyond $100 \mu \mathrm{m}$, if interpreted as dust emission, would imply a very cold temperature, and it is possible that such excesses are due to contamination from background galaxies (Krivov et al. 2013; Gáspár \& Rieke 2014). From Trilling et al. (2007), HD 46273 and HD 173608 were both deemed in that paper to have MIPS $70 \mu \mathrm{m}$ excesses, but in both cases the photometry in that band, while a little high (2$3 \sigma)$, is considerably below our excess threshold. Additionally, both were reobserved as part of the OT2_gkennedy_2 survey, and the PACS $70 \mu \mathrm{m}$ photometry is in line with the model for the stellar photosphere. Finally, while the Rodriguez \& Zuckerman (2012) systems were not included in Fig. 8, it can be seen from Fig. 5 that none of these lie in the separation range of interest (and satisfy our excess criterion) either. There are two systems in our sample from that paper that fall in that separation range: HD 56986 and HD 73752. Both have slightly high (2-3o) IRAS $60 \mu \mathrm{m}$ fluxes and consequently were previously thought to host discs - but also MIPS $70 \mu \mathrm{m}$ and PACS $100 \mu \mathrm{m}$ fluxes that agree well with the stellar model. We are therefore satisfied that no excesses have been 'missed' in the separation range in question, and that there is a genuine absence of debris discs there.

It is also instructive to examine the disc detection fraction as a function of stellar separation. This is illustrated in Fig. 10, where the separation bins were chosen as $a<25 \mathrm{au}, 25 \mathrm{au}<a<135 \mathrm{au}$, and $a>135$ au to highlight the lack of discs in medium separation systems outlined above; the error bars show binomial uncertainties. This figure shows that discs appear to be significantly more common in wide binaries, with a detection rate of $\frac{18}{95}=19_{-3}^{+5}$ per cent, than in those with close orbits, at $\frac{18}{231}=8_{-1}^{+2}$ per cent. Considering only the systems with well-known orbits gives consistent results: 70 known orbit components lie in the closest semimajor axis bin; this includes all four with discs, giving a detection rate in this bin of $6_{-2}^{+4}$ per cent. The remaining 17 unbiased known orbit components 
lie in the medium separation bin. Our overall disc detection rate for all unbiased systems across all separations is $9_{-1}^{+2}$ per cent, consistent with the $11 \pm 3$ per cent derived by Rodriguez et al. (2015). The detection rates per spectral type are $22_{-5}^{+7}$ per cent for A stars, $11_{-2}^{+3}$ per cent for $\mathrm{F}$ stars, $5_{-1}^{+3}$ per cent for $\mathrm{G}$ stars, and $7_{-2}^{+4}$ per cent for $\mathrm{K}$ stars, with no discs detected around $\mathrm{M}$ stars; these results are again comparable with those of Rodriguez et al. (2015).

\section{DISCUSSION}

In this section, we begin with a discussion of how our findings relate to the results of studies of protoplanetary discs and planets in binary systems. We then turn our attention to the issue of warm excesses in close binaries discussed by Matranga et al. (2010), and discuss what the OT1_jdrake01_1 data add to our understanding of such systems.

\subsection{Relation to protoplanetary discs}

Perhaps the most important result of this paper is the apparent lack of debris discs in systems with stellar separation $a$ between $\sim 25$ and $135 \mathrm{au}$. This range of separations corresponds well to the typical disc radii that we derive, with the lower and upper quartiles of $r_{\text {disc }}$ for our full sample being $\sim 30$ and 140 au. Thus, the natural conclusion is that the lack of discs in medium separation binaries is a result of these systems clearing out their circumstellar or circumbinary material via dynamical perturbations. As detailed in Section 1, similar results have been hinted at in previous work (e.g. Trilling et al. 2007), though not in a statistically significant way.

The properties of protoplanetary discs in binary systems are also known to be dependent on stellar separation. It has been well established that the $\sim$ mm fluxes of pre-main-sequence (PMS) binaries are significantly lower for systems with $1 \lesssim a \lesssim 100$ au than for $a \gtrsim 100$ au (e.g. Osterloh \& Beckwith 1995; Jensen, Mathieu \& Fuller 1996; Andrews \& Williams 2005; Duchêne 2010; Kraus et al. 2012). This implies that systems with a separation comparable to typical disc sizes tend to have a lower protoplanetary disc mass, likely due to the dynamical influence of the binary companion. Cieza et al. (2009) similarly found that PMS systems with 1 $\lesssim a \lesssim 40$ au are significantly less likely to have an excess at $8 \mu \mathrm{m}$ (with the shorter wavelength probing the innermost part of a protoplanetary disc) than those with $40 \lesssim a \lesssim 400$ au. Since protoplanetary discs are the precursors of debris discs, in that the planetesimals comprising a debris disc are formed from material in the protoplanetary disc, we would expect to find a dearth of detectable debris discs for separations of order $\sim 1-100 \mathrm{au}$; this is indeed similar to our conclusion.

If the properties of the debris discs we are studying are indeed influenced by stellar perturbations to their primordial protoplanetary discs, one might also expect circumbinary debris discs (for which truncation occurs from the inner edge) to have larger radii on average than circumstellar debris discs in binary systems (for which truncation occurs from the outer edge). We find that the median of $r_{\text {disc }}$ is 75 au for our $a<25$ au systems, compared with 55 au for our $a>135$ au systems, consistent with this idea. This may be an interesting result, however, given the relatively small number of discs it is not statistically significant, with a KS test on the disc radii of the close and wide binaries returning a $p$-value of 0.84 .

We also found that the dust detection rate for the widest binaries in our sample, $19_{-3}^{+5}$ per cent, is comparable with single star rates
(Su et al. 2006; Hillenbrand et al. 2008; Trilling et al. 2008; Eiroa et al. 2013; Sierchio et al. 2014; Thureau et al. 2014; Sibthorpe et al. 2018), while for the closest binaries debris discs are around half as common, with a detection rate of $8_{-1}^{+2}$ per cent. Statistical studies of discs around PMS binaries have not generally included systems with $a \lesssim 1$ au since PMS multiplicity surveys at such close separations are highly incomplete; we note, however, that at least several PMS binaries with $a \lesssim 1$ au are known to have high submillimetre fluxes (e.g. Mathieu et al. 1995, 1997; Kennedy et al. 2019). Thus, it is not known whether protoplanetary disc properties in $a \lesssim 1$ au binaries are significantly different to those in $a \gtrsim 100$ au binaries, and the reason for the lower debris disc incidence that we find in close binaries is not clear. It could be the case that close binaries tend to have lower protoplanetary disc masses than wide binaries, and thus a reduced ability to form planetesimals. Alternatively, it is possible that close binaries do form planetesimals as efficiently as wide binaries, but are able to drive faster collisional evolution of the resulting debris discs.

To summarize, medium separation binaries are lacking in circumstellar or circumbinary material from early in their lives. It appears that our conclusion that those on the main sequence lack detectable levels of dust is not predominantly a result of the dynamical evolution of planetesimals in the debris disc phase, but follows naturally from the lower masses of the primordial discs from which planetesimals form. Our result is ultimately due to the fact that the dynamical influence of the binary is important when $a$ is similar to typical values of $r_{\text {disc }}$, but this influence leaves its observable signature on systems at an early stage.

\subsection{Relation to planets}

The occurrence rate of planets in binaries is a strong function of stellar separation. Numerous studies have demonstrated that the separations of known planet-hosting binaries are significantly larger than those of field binaries, with the conclusion being that planet formation appears to be inhibited in systems closer than $\sim 10-100$ au (e.g. Bergfors et al. 2013; Wang et al. 2014; Kraus et al. 2016; Fontanive et al. 2019). Since the detection of a debris disc in a given system indicates that planetesimals were able to form therein, our result that discs are significantly less common for systems in the closest bin of Fig. 10 than those in the widest bin may lead to the similar conclusion that planetesimal formation is hindered in systems with $a<25$ au (see also Section 5.1).

Another potentially interesting result from the literature relates to even closer binaries: Welsh et al. (2014) noted that of the eight systems discovered by Kepler to host circumbinary planets, none have binary periods shorter than $\sim 7 \mathrm{~d}$, contrasting with the fact that the periods of most Kepler eclipsing binaries are below $1 \mathrm{~d}$. Fabrycky \& Tremaine (2007) explored the idea that the closest binaries (those with periods less than $\sim 10 \mathrm{~d}$, corresponding to semimajor axes below $\sim 0.1$ au for Sun-like stars) are initially much wider, then have their orbits shrunk by Kozai cycles induced by a tertiary stellar companion combined with tidal friction. Martin, Mazeh \& Fabrycky (2015) suggested that this process could explain the apparent lack of circumbinary planets around very close binaries, since the third star would also perturb any circumbinary material, hindering planet formation and survival.

From Fig. 8, it is clear that there is no 'cut-off' separation below which no debris discs are found, in contrast with the circumbinary Kepler planet results. In fact, the disc detection rate for the $\mathrm{OT} 1_{-}$j drake01_1 sample of very close binaries is consistent with that for all systems with $a<25$ au (see Section 5.3). This may 
suggest that the mechanism of Kozai cycles with tidal friction is not responsible for producing all (or the overwhelming majority) of the close binaries in our sample, as we would generally expect the planetesimals comprising debris discs to be cleared out in systems where this process has taken place. Thus, we expect that planets do indeed exist around some very close binaries and that such systems should be detected given a larger sample of circumbinary planets in the future.

\subsection{Infrared emission from close binaries}

As noted earlier in this paper, Matranga et al. (2010) studied a sample of 10 close binaries and found warm infrared excesses in three of them using $24 \mu \mathrm{m}$ photometry from MIPS and shorter wavelength photometry from Spitzer's Infrared Array Camera (Fazio et al. 2004). They interpreted these warm excesses as possible emission from dust produced in collisions between planetary bodies. As such dust is not being continuously replenished, it should be relatively short-lived, and to detect it would require observing a system at a special point in its evolution. It would thus be somewhat surprising if 30 per cent of their sample were genuinely undergoing such an event at the time of observation. Our sample contains 50 systems with PACS data from the OT1_j drake 01_1 survey, which was designed as a longer wavelength follow-up to Matranga et al. (2010), and in this subsection we consider whether these data offer any insight into the nature of the infrared emission from close binaries.

The OT1_jdrake01_1 systems have a median separation of $0.05 \mathrm{au}$ (or a median period of $\sim 4 \mathrm{~d}$ ), and constitute a sample of the very closest binaries in our study. According to the criteria of Section 3, we detect formal $70 \mu \mathrm{m}$ excesses in $\frac{4}{50}=8_{-2}^{+6}$ per cent of these systems. While one of these excesses is the exceptionally warm HD 21242 (UX Ari; see Section 4.3), it is clear from Fig. 4 that very close binaries do not generally have unusually warm dust temperatures; if this system is discounted then the detection rate is $6_{-2}^{+5}$ per cent. Regardless of whether HD 21242 is included, the OT1_jdrake01_1 detection rate is in good agreement with the $8_{-1}^{+2}$ per cent that we derived for the $a<25$ au systems in Section 4.4, which suggests that even if there is some process in the closest binaries that tends to produce warm dust - e.g. resonance sweeping caused by secular shrinkage of the binary orbit due to tidal interactions, as proposed by Matranga et al. (2010) - it does not significantly affect the levels of Kuiper belt-like cool dust in these systems.

None the less, recall from Section 3 that we do find a striking difference between the significance distributions of PACS $70 \mu \mathrm{m}$ photometry for the OT1_jdrake01_1 systems and the remainder of our sample, with the OT1_jdrake01_1 fluxes appearing systematically higher than expected. To determine whether this offset is related to the apparent warm excesses found by Matranga et al. (2010), we can examine a colour-colour diagram, as shown in Fig. 11. In this figure, the horizontal axis shows the magnitude in the WISE $12 \mu \mathrm{m}$ band minus the $\sim 22 \mu \mathrm{m}$ magnitude, where we used WISE $22 \mu \mathrm{m}$ photometry where available and MIPS $24 \mu \mathrm{m}$ otherwise. Similarly, the vertical axis shows $\sim 22 \mu \mathrm{m}$ magnitude minus far-infrared magnitude, for which we use PACS $70 \mu$ m fluxes where available, and MIPS $70 \mu \mathrm{m}$ preferentially to PACS $100 \mu \mathrm{m}$ where not. Each point represents a system in our sample, with the OT1_jdrake01_1 systems highlighted as the darker points. Systems that we deem to host a disc are plotted with the larger symbols. The uncertainties in both colours, which depend on the fractional uncertainties in the fluxes, are found to have narrow

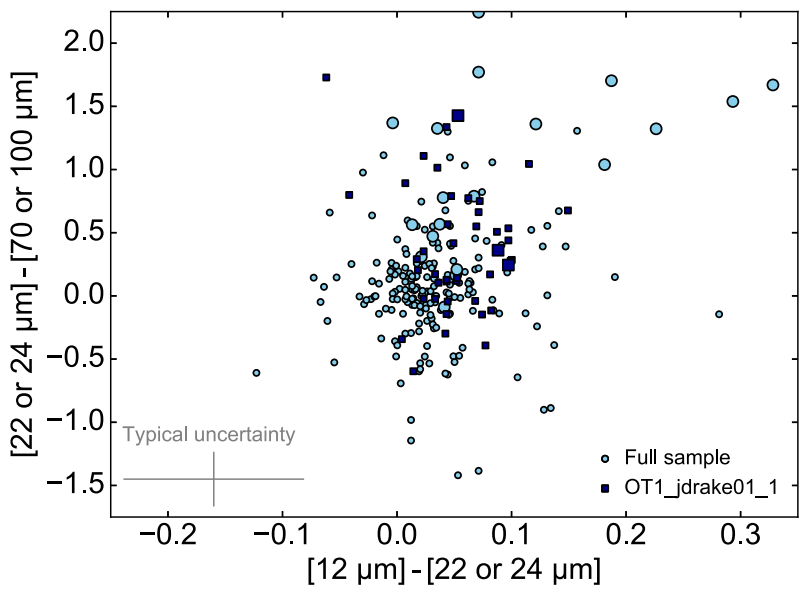

Figure 11. A colour-colour diagram for our full sample, with systems from the OT1_jdrake01_1 survey highlighted. The horizontal axis shows WISE $12 \mu \mathrm{m}$ magnitude minus WISE $22 \mu \mathrm{m}$ magnitude where available and MIPS $24 \mu \mathrm{m}$ otherwise. The vertical axis shows 22 or $24 \mu \mathrm{m}$ magnitude minus far-infrared magnitude, where for far-infrared fluxes we preferentially used PACS $70 \mu \mathrm{m}$ over MIPS $70 \mu \mathrm{m}$ over PACS $100 \mu \mathrm{m}$. Systems with debris discs are plotted with the larger symbols. The error bars show the median uncertainties in each colour. Systems towards the right (or the top) have SEDs that decrease less steeply than Rayleigh-Jeans between 12 and $\sim 22 \mu \mathrm{m}$ ( or $\sim 22$ and $\sim 70 \mu \mathrm{m}$ ), while the SEDs of those towards the left (or the bottom) decrease more steeply. The OT1_j drake01_1 sample appears to be systematically offset above and to the right of the rest of the systems.

distributions; the typical error bars showing the median uncertainties are displayed in the figure.

To interpret Fig. 11 physically, note that systems with purely stellar SEDs have roughly equal magnitudes everywhere in their Rayleigh-Jeans tails and thus lie near the origin. Systems lying further to the right have fluxes that decrease less steeply than the Rayleigh-Jeans tail of a stellar SED between 12 and $\sim 22 \mu \mathrm{m}$; similarly for the fluxes of systems further towards the top between $\sim 22$ and $\sim 70 \mu \mathrm{m}$. Conversely, systems below or to the left of the origin have more steeply declining fluxes over the relevant wavelength range. Not all systems are visible in the plot; the brightest excesses lie outside the range shown.

In Fig. 11, it can be seen that as a population, the OT1_jdrake01_1 systems are concentrated above and to the right of the rest of the sample. That is, these systems tend to have SEDs that are declining less steeply than for a pure Rayleigh-Jeans tail both from 12 to $\sim 22 \mu \mathrm{m}$ and from $\sim 22$ to $\sim 70 \mu \mathrm{m}$. This situation can be contrasted with the case of HD 69830, a single star with an excess at $\sim 22 \mu \mathrm{m}$ (Beichman et al. 2005) but not at $\sim 70 \mu \mathrm{m}$ (Marshall et al. 2014). The excess of HD 69830 has been interpreted as due to debris from a destructive collision of a $\sim 30 \mathrm{~km}$-sized asteroid (Beichman et al. 2011), similar to the scenario proposed by Matranga et al. (2010) to explain the infrared emission from close binaries. Its excess peaks at around $20 \mu \mathrm{m}$, with its SED falling less steeply than Rayleigh-Jeans from 12 to $\sim 22 \mu \mathrm{m}$, then more steeply from $\sim 22 \mu \mathrm{m}$ to $\sim 70 \mu \mathrm{m}$, and would thus lie to the right and below the bulk population in Fig. 11. We would expect the SEDs of other systems experiencing such a collision to have similar shapes to that of HD 69830, peaking at a few tens of $\mu \mathrm{m}$ and then declining more steeply than a blackbody. This is because a collision between two massive bodies would be expected to produce an excess of small grains compared with a steady-state debris disc, meaning that in such cases $\lambda_{0}$, the wavelength beyond which the 
SED decreases more steeply (see Section 3), should be relatively small. In addition, the presence of silicate emission features at $\sim 10$ and $20 \mu \mathrm{m}$ (see e.g. Matthews et al. 2014) could act to steepen the SED between mid- and far-infrared wavelengths. We note that of the OT1_jdrake01_1 sample, only HD 8997, HD 118216, and HIP 3362 have Spitzer IRS spectra, but none of these show the silicate features that we might expect to see if an abundance of small grains was present.

In fact, the excesses proposed by Matranga et al. (2010), being exceptionally hot (1000-2000 K), peak at even shorter wavelengths than for HD $69830(\sim 5 \mu \mathrm{m})$. We might expect the SEDs of systems with such hot excesses to be falling more steeply than RayleighJeans even from 12 to $\sim 22 \mu \mathrm{m}$, placing them towards the left-hand side of Fig. 11. In either case, while it appears from Fig. 11 (as well as Figs 2 and 3) that the SEDs of the OT1_jdrake01_1 sample are indeed somehow different from our bulk sample, their positions in this figure do not seem to support the idea that massive collisions are much more common for close binaries than for wider binaries or single stars.

The above discussion is based on the assumption that the dust is optically thin, and that its emission is therefore well described by the modified blackbody function defined in Section 3. The $\mathrm{OT} 1_{-}$jdrake01_1 SEDs may in fact be consistent with optically thick dust, as the emission of such dust is better described by an unmodified blackbody. However, the short collisional lifetime of warm dust would still be an issue, perhaps even more so if the dust were required to be present at high enough density to be optically thick.

To summarize, the expected transience of warm dust from giant collision events, combined with the shapes of the infrared spectra of the OT1_jdrake01_1 systems as determined from a colourcolour diagram, indicate that further work is required to understand the true nature of the infrared emission from close binaries. It appears that such binaries are subject to some unknown systematic effect causing larger than expected fluxes in the Rayleigh-Jeans tails of their SEDs, which could cause warm dust to appear more common than it really is.

\section{CONCLUSIONS}

We have assembled a sample of 341 binary and multiple star systems, and modelled their SEDs to identify and characterize debris discs in these systems. Our sample, defined in Section 2, is a combination of the systems studied by Trilling et al. (2007), Rodriguez \& Zuckerman (2012), and Rodriguez et al. (2015), and the systems targeted by the Herschel programmes OT2_gkennedy_2 (a survey of visual binaries with well-known orbits) and OT1_jdrake01_1 (a survey of close binaries).

To each SED, we fitted a model with both a stellar component and a modified blackbody dust component. As outlined in Section 3, we defined an infrared excess criterion based on the measured distributions of photometric significances, such that systematic effects and underestimated or overestimated uncertainties are accounted for. Systems with significant excesses in the MIPS $70 \mu \mathrm{m}$, PACS $70 \mu \mathrm{m}$, and/or PACS $100 \mu \mathrm{m}$ bands were considered to host a debris disc. In Section 4.1, we found that there are 38 such systems. We converted the dust temperatures from SED fitting into blackbody radii $r_{\mathrm{bb}}$, then corrected these for the effect of the inefficient emission of small grains using the prescription of Pawellek \& Krivov (2015). The resulting radii $r_{\text {disc }}$ were used in Section 4.2 to assess the dynamical stability of the discs according to the criteria of Holman \& Wiegert (1999). Nine of the discs appear based on a simple analysis of their radii and host binary separations to be unstable, and we discussed each of these systems in detail in Section 4.3. Considering the expected short lifetime of unstable dust, we are hesitant to conclude that the apparent instability is real. Uncertainty in the true disc radius due to scatter in the correction factor from $r_{\mathrm{bb}}$ to $r_{\text {disc }}$, uncertainty in the boundary of the unstable region (which depends on the binary eccentricity and mass ratio), and projection of the binary semimajor axis could be responsible for stable systems appearing to be unstable.

We found in Section 4.4 that the separations of the disc-bearing and disc-free systems are drawn from different distributions at a confidence level of 99.4 percent, higher than the 91 percent found previously by Rodriguez et al. (2015). We thus concluded that the binary separation $a$ influences the presence of detectable levels of debris in a statistically significant way, in contrast with the conclusion of Rodriguez et al. (2015). The disc detection rate is $19_{-3}^{+5}$ per cent for systems with $a>135 \mathrm{au}$, comparable with published results for single stars. Only $8_{-1}^{+2}$ per cent of those with $a<25$ au have a detectable disc, which suggests that either planetesimal formation is inhibited in binaries closer than a few tens of au, or that such systems are able to drive faster collisional evolution in their debris discs than wide binaries. We did not find any significant infrared excesses in systems with 25 au $<a<135$ au. Our main conclusion is thus that there is a lack of debris discs in medium separation systems.

We compared our results with studies of PMS binaries in Section 5.1, concluding that the lack of debris discs in medium separation systems is likely a result of their precursors, protoplanetary discs, being dynamically cleared of material when the stellar separation is similar to typical disc radii. In Section 5.2, we discussed how our conclusion that binaries with $a<25$ au are less likely to host a detectable debris disc relates to the results of studies of known planet-hosting binaries, which have suggested that planet formation is hindered in binaries closer than a few tens of au. We then considered the non-detection by Kepler of circumbinary planets in very short-period binaries, and suggested that our detection of discs in such systems implies that future observations should in fact find such planets. Finally, in Section 5.3 we explored the idea of frequent planetary collisions in close binaries proposed by Matranga et al. (2010), concluding that the closest binaries in our sample do not tend to have the colours we would expect in such a scenario.

\section{ACKNOWLEDGEMENTS}

We thank the reviewer for a report that helped to improve the quality of the paper. BY acknowledges the support of a Science and Technology Facilities Council studentship. GMK is supported by the Royal Society as a Royal Society University Research Fellow.

\section{REFERENCES}

Allard F., Homeier D., Freytag B., 2012, Phil. Trans. R. Soc. A, 370, 2765 Andrews S. M., Williams J. P., 2005, ApJ, 631, 1134

Armstrong D. J., Osborn H. P., Brown D. J. A., Faedi F., Gómez Maqueo Chew Y., Martin D. V., Pollacco D., Udry S., 2014, MNRAS, 444, 1873 Asplund M., Grevesse N., Sauval A. J., Scott P., 2009, ARA\&A, 47, 481 Aumann H. H. et al., 1984, ApJ, 278, L23

Backman D. E., Paresce F., 1993, in Levy E. H., Lunine J. I., eds, Protostars and Planets III. Univ. Arizona Press, Tucson, AZ, p. 1253

Balona L. A., 1987, South African Astronomical Observatory Circular, 11, 1

Beichman C. A. et al., 2005, ApJ, 626, 1061

Beichman C. A. et al., 2011, ApJ, 743, 85 
Bergfors C. et al., 2013, MNRAS, 428, 182

Burgasser A. J., Kirkpatrick J. D., Reid I. N., Brown M. E., Miskey C. L., Gizis J. E., 2003, ApJ, 586, 512

Burns J. A., Lamy P. L., Soter S., 1979, Icarus, 40, 1

Cieza L. A. et al., 2009, ApJ, 696, L84

Costa E., Méndez R. A., Jao W.-C., Henry T. J., Subasavage J. P., Brown M. A., Ianna P. A., Bartlett J., 2005, AJ, 130, 337

Dominik C., Decin G., 2003, ApJ, 598, 626

Drake J., 2010, OT1_jdrake01_1: Close Binaries with Infrared Excess: Destroyers of Worlds? Herschel Space Observatory Proposal, id. 941

Duchêne G., 2010, ApJ, 709, L114

Duchêne G., Kraus A., 2013, ARA\&A, 51, 269

Duemmler R., Aarum V., 2001, A\&A, 370, 974

Duquennoy A., Mayor M., 1991, A\&A, 248, 485

Eggleton P. P., Tokovinin A. A., 2008, MNRAS, 389, 869

Eiroa C. et al., 2010, A\&A, 518, L131

Eiroa C. et al., 2013, A\&A, 555, A11

Fabrycky D., Tremaine S., 2007, ApJ, 669, 1298

Fazio G. G. et al., 2004, ApJS, 154, 10

Feroz F., Hobson M. P., Bridges M., 2009, MNRAS, 398, 1601

Fontanive C., Rice K., Bonavita M., Lopez E., Mužić K., Biller B., 2019, MNRAS, 485, 4967

Foreman-Mackey D., Hogg D. W., Lang D., Goodman J., 2013, PASP, 125, 306

Frasca A., Guillout P., Marilli E., Freire Ferrero R., Biazzo K., Klutsch A., 2006, A\&A, 454, 301

Fruchter A. S., Hook R. N., 2002, PASP, 114, 144

Gaia Collaboration, 2018, A\&A, 616, A1

Gáspár A., Rieke G. H., 2014, ApJ, 784, 33

Geyer D. W., Harrington R. S., Worley C. E., 1988, AJ, 95, 1841

Harrington R. S., Dahn C. C., 1980, AJ, 85, 454

Hartkopf W. I., Mason B. D., Worley C. E., 2001, AJ, 122, 3472

Henry T. J., Jao W.-C., Subasavage J. P., Beaulieu T. D., Ianna P. A., Costa E., Méndez R. A., 2006, AJ, 132, 2360

Hillenbrand L. A. et al., 2008, ApJ, 677, 630

Holland W. S. et al., 2017, MNRAS, 470, 3606

Holman M. J., Wiegert P. A., 1999, AJ, 117, 621

Horch E. P., Howell S. B., Everett M. E., Ciardi D. R., 2014, ApJ, 795, 60

Houck J. R. et al., 2004, ApJS, 154, 18

Hughes A. M., Duchêne G., Matthews B. C., 2018, ARA\&A, 56, 541

Ishihara D. et al., 2010, A\&A, 514, A1

Jensen E. L. N., Mathieu R. D., Fuller G. A., 1996, ApJ, 458, 312

Johansen A., Blum J., Tanaka H., Ormel C., Bizzarro M., Rickman H., 2014, in Beuther H., Klessen R., S., Dullemond C. P., Henning T., eds, Protostars and Planets VI. Univ. Arizona Press, Tucson, AZ, p. 547

Kennedy G. M., Wyatt M. C., 2014, MNRAS, 444, 3164

Kennedy G. M. et al., 2012a, MNRAS, 421, 2264

Kennedy G. M., Wyatt M. C., Sibthorpe B., Phillips N. M., Matthews B. C., Greaves J. S., 2012b, MNRAS, 426, 2115

Kennedy G. M. et al., 2019, Nat. Astron., 3, 230

Kiraga M., 2012, Acta Astron., 62, 67

Kraus A. L., Ireland M. J., Hillenbrand L. A., Martinache F., 2012, ApJ, 745,19

Kraus A. L., Ireland M. J., Huber D., Mann A. W., Dupuy T. J., 2016, AJ, 152,8

Krist J. E. et al., 2010, AJ, 140, 1051

Krivov A. V. et al., 2013, ApJ, 772, 32

Lebreton J. et al., 2012, A\&A, 539, A17

Lindegren L. et al., 2018, A\&A, 616, A2

Lisse C. M., Beichman C. A., Bryden G., Wyatt M. C., 2007, ApJ, 658, 584

Mannings V., Barlow M. J., 1998, ApJ, 497, 330

Marshall J. P. et al., 2011, A\&A, 529, A117

Marshall J. P. et al., 2014, A\&A, 565, A15

Martin D. V., Mazeh T., Fabrycky D. C., 2015, MNRAS, 453, 3554

Mathieu R. D., Adams F. C., Fuller G. A., Jensen E. L. N., Koerner D. W., Sargent A. I., 1995, AJ, 109, 2655

Mathieu R. D., Stassun K., Basri G., Jensen E. L. N., Johns-Krull C. M., Valenti J. A., Hartmann L. W., 1997, AJ, 113, 1841
Matranga M., Drake J. J., Kashyap V. L., Marengo M., Kuchner M. J., 2010, ApJ, 720, L164

Matthews B. C. et al., 2010, A\&A, 518, L135

Matthews B. C., Krivov A. V., Wyatt M. C., Bryden G., Eiroa C., 2014, in Beuther H., Klessen R. S., Dullemond C. P., Henning T., eds, Protostars and Planets VI. Univ. Arizona Press, Tucson, AZ, p. 521

Murray C. D., Dermott S. F., 1998, Solar System Dynamics. Cambridge Univ. Press, Cambridge

Neuhäuser R., Ginski C., Schmidt T. O. B., Mugrauer M., 2011, MNRAS, 416, 1430

Osterloh M., Beckwith S. V. W., 1995, ApJ, 439, 288

Oudmaijer R. D., van der Veen W. E. C. J., Waters L. B. F. M., Trams N. R., Waelkens C., Engelsman E., 1992, A\&AS, 96, 625

Pawellek N., Krivov A. V., 2015, MNRAS, 454, 3207

Phillips N. M., 2011, PhD thesis, Univ. Edinburgh

Poglitsch A. et al., 2010, A\&A, 518, L2

Pourbaix D. et al., 2004, A\&A, 424, 727

Raghavan D. et al., 2010, ApJS, 190, 1

Rhee J. H., Song I., Zuckerman B., 2008, ApJ, 675, 777

Rieke G. H. et al., 2004, ApJS, 154, 25

Rodriguez D. R., Zuckerman B., 2012, ApJ, 745, 147

Rodriguez D. R., Duchêne G., Tom H., Kennedy G. M., Matthews B., Greaves J., Butner H., 2015, MNRAS, 449, 3160

Sibthorpe B., Kennedy G. M., Wyatt M. C., Lestrade J.-F., Greaves J. S., Matthews B. C., Duchêne G., 2018, MNRAS, 475, 3046

Sierchio J. M., Rieke G. H., Su K. Y. L., Gáspár A., 2014, ApJ, 785, 33

Smith R., Churcher L. J., Wyatt M. C., Moerchen M. M., Telesco C. M., 2009, A\&A, 493, 299

Song I., Zuckerman B., Weinberger A. J., Becklin E. E., 2005, Nature, 436, 363

Su K. Y. L. et al., 2006, ApJ, 653, 675

Thébault P., Marzari F., Augereau J.-C., 2010, A\&A, 524, A13

Thureau N. D. et al., 2014, MNRAS, 445, 2558

Tokovinin A., 2018, ApJS, 235, 6

Torres G., Andersen J., Giménez A., 2010, A\&AR, 18, 67

Trilling D. E. et al., 2007, ApJ, 658, 1289

Trilling D. E. et al., 2008, ApJ, 674, 1086

van Altena W. F., Lee J. T., Hoffleit E. D., 1995, The General Catalogue of Trigonometric [stellar] Parallaxes. Yale University Observatory, New Haven, CT

van Leeuwen F., 2007, A\&A, 474, 653

Wang J., Xie J.-W., Barclay T., Fischer D. A., 2014, ApJ, 783, 4

Welsh W. F., Orosz J. A., Carter J. A., Fabrycky D. C., 2014, in Haghighipour N., ed., Proc. IAU Symp. 293, Formation, Detection, and Characterization of Extrasolar Habitable Planets. Cambridge University Press, Cambridge. p. 125

Wiegert J., Faramaz V., Cruz-Saenz de Miera F., 2016, MNRAS, 462, 1735

Wright E. L. et al., 2010, AJ, 140, 1868

Wyatt M. C., 2005, A\&A, 433, 1007

Wyatt M. C., 2008, ARA\&A, 46, 339

Wyatt M. C., Smith R., Greaves J. S., Beichman C. A., Bryden G., Lisse C. M., 2007, ApJ, 658, 569

Wyatt M. C., Panić O., Kennedy G. M., Matrà L., 2015, Ap\&SS, 357, 103

\section{SUPPORTING INFORMATION}

Supplementary data are available at MNRAS online.

Table 1. Information on the orbits of the binaries and multiple systems in our sample.

Table 2. Photometry in the MIPS $70 \mu \mathrm{m}$, PACS $70 \mu \mathrm{m}$, and PACS $100 \mu \mathrm{m}$ bands for all systems in our sample.

Table 3. Results of SED fitting for the 38 systems we find to have a significant infrared excess.

Please note: Oxford University Press is not responsible for the content or functionality of any supporting materials supplied by 
the authors. Any queries (other than missing material) should be directed to the corresponding author for the article.

\section{APPENDIX: RESOLVED IMAGE MODELLING OF HD 95698}

A single disc - that of HD 95698 - was resolved in the OT2_gkennedy_2 observations. Here, we outline the method we used to model this disc, and present the results of this modelling.

A cut-out of the $70 \mu \mathrm{m}$ image of HD 95698 from that survey is shown in the leftmost panel of Fig. A1. The centre left-hand panel of that figure shows the residuals following a PSF subtraction. We use an observation of the Herschel calibration star HD 164058 (gamma Dra), normalized and rotated to the appropriate orientation, as a PSF. For the subtraction, we scaled the PSF such that its peak intensity is equal to the peak intensity in the image. We allowed the PSF to have a variable offset since the Herschel pointing is not perfect, and the residuals shown correspond to the offset that gives the best fit. A prominent ring of emission remains, indicating that the disc in this system is indeed resolved.

One pixel in the image covers 1.6 arcsec, and given that the system is at a distance of $57.4 \mathrm{pc}$ (van Leeuwen 2007) this corresponds to 92 au per pixel. Given the low spatial resolution of the data, we generate our model images on a higher resolution grid with $13 \times 13$ smaller pixels per PACS pixel. Our model for the resolved disc is axisymmetric and optically thin, and assumes that the surface density is proportional to $r^{-1}$, where $r$ is the distance from the centre of mass of the binary, and thus that the surface brightness is proportional to $r^{-1.5}$ (assuming that the dust temperature at a distance $r$ is proportional to $r^{-0.5}$, as is the case for blackbody grains). We also tried an alternative model in which the surface brightness varies as $r^{-\alpha}$, with $\alpha$ as a free parameter. However, we found that $\alpha$ was poorly constrained, with the model preferring to have unphysically steep profiles and unphysically large outer disc radii such that the disc emission drops below the noise floor. Thus, we ultimately decided to fix $\alpha$ at a value of 1.5 , which we consider to be physically reasonable.

The model has as free parameters the total flux of the resolved disc $F_{\text {res }}$, inner and outer edges $r_{1}$ and $r_{2}$, inclination $I$, and position angle of the major axis $\theta$ (measured anticlockwise from north). Our SED fitting gives the expected stellar flux as $5.372 \mathrm{mJy}$. It is known that
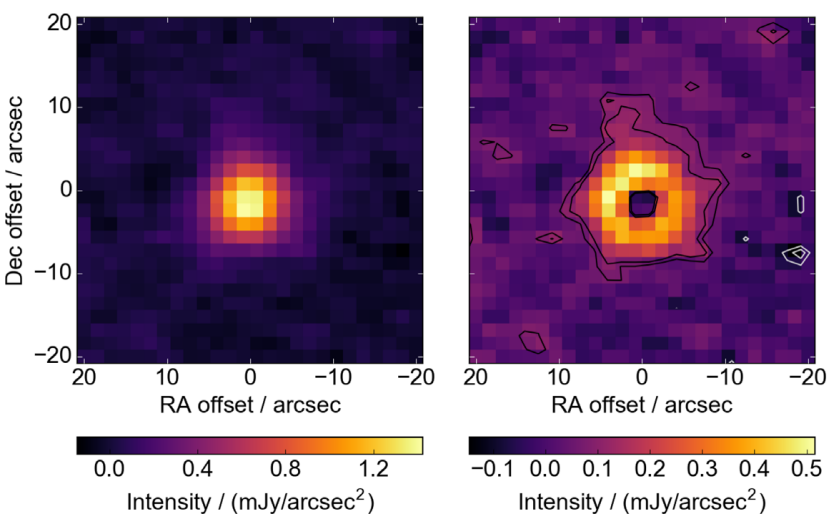

for PACS data the flux obtained from PSF fitting will underestimate the true value as some flux is lost in the wings of the PSF during image processing; at $70 \mu \mathrm{m}$ this underestimation is by a factor of around 1.16 (Kennedy et al. 2012a). Thus, at the pixel in the centre of the disc we set the flux to $F_{\text {star }}+F_{\text {unres }}$, where $F_{\text {star }}=\frac{5.372}{1.16} \mathrm{mJy}$, and $F_{\text {unres }}$ is a free parameter allowing for an unresolved component of the excess. Including an arbitrary 2D offset - which is unrelated to the properties of the disc but accounts for non-perfect Herschel pointing - there are therefore eight free parameters in total.

After resampling the PSF to the higher resolution model grid, we convolve the model with the PSF; the resulting synthetic image is then resampled back to the PACS pixel size for comparison with the observations. We calculate the goodness of fit $\chi^{2}-$ which is unrelated to the significance $\chi$ defined in equation (1) - as follows:

$\chi^{2}=\sum_{i} \frac{\left(F_{\mathrm{obs}, i}-F_{\mathrm{model}, i}\right)^{2}}{\sigma^{2}}$.

Here, $F_{\mathrm{obs}, i}$ and $F_{\text {model }, i}$ are the observed and model fluxes in pixel $i$, and $\sigma$ is the corresponding uncertainty. We calculate the sum over a $26 \times 26$ pixel box centred on the star. To estimate the appropriate value of $\sigma$, we first took the root mean square (RMS) of the flux per pixel in an empty $20 \times 20$ pixel patch of sky near the star, giving $0.097 \mathrm{mJy}$. As outlined in Kennedy et al. (2012a), the noise in a PACS image is correlated, and this effect can be accounted for by scaling the RMS by the factor defined in equation (9) of Fruchter \& Hook (2002). The ratio of natural to actual pixel scales in the image we are modelling is $\frac{3.2^{\prime \prime}}{1.6^{\prime \prime}}=2$, which leads to the result $\sigma=0.23 \mathrm{mJy}$. To find the best-fitting model, we first estimated the parameters that minimize $\chi^{2}$ using differential evolution, an algorithm designed to find the global minimum of a function, avoiding the potential issue of instead returning a local minimum. To quantify the posterior distributions of the model parameters, we then ran the Markov Chain Monte Carlo algorithm implemented by Foreman-Mackey et al. (2013), with a likelihood function $e^{-\chi^{2} / 2}$. We used 250 walkers initialized close to the previously identified minimum $\chi^{2}$ solution, and ran the algorithm for 1000 steps. Inspection of the individual chains led us to discard the first 700 steps as the burn-in period.

The resulting parameters can by quantified by the median values of the posterior distributions, with uncertainties corresponding to the 16th and 84th percentiles: $F_{\text {unres }}=19_{-13}^{+14} \mathrm{mJy}, F_{\text {res }}=99_{-15}^{+14} \mathrm{mJy}$,
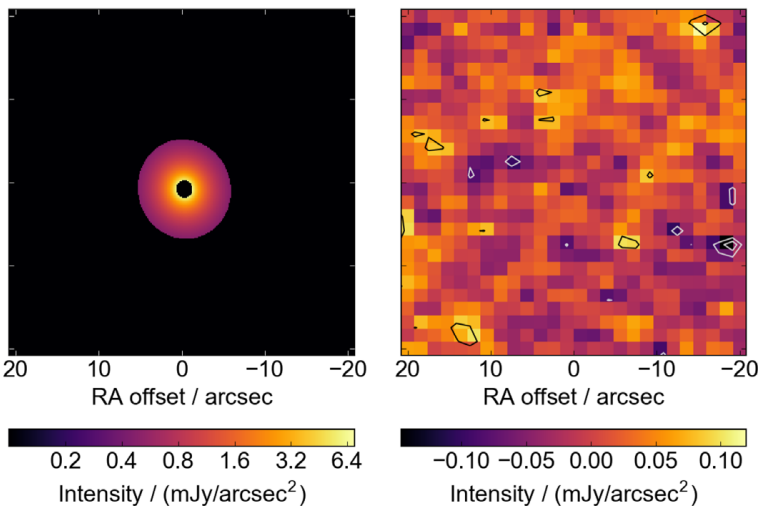

Figure A1. Far left: cut-out of the PACS $70 \mu \mathrm{m}$ image of HD 95698 from the OT2_gkennedy_2 survey. Centre left: residuals following a PSF subtraction, with the PSF normalized to the brightest pixel in the observed image. The contours show \pm 2 and 3 times the root-mean-square intensity. The ring of significant emission that remains indicates that the disc is resolved. Centre right: unconvolved high-resolution image of the minimum $\chi^{2}$ model as determined using the Markov Chain Monte Carlo method. For clarity, we do not plot the central pixel containing the stellar and unresolved fluxes, and the colour scale is logarithmic with the background level arbitrarily set to $0.1 \mathrm{mJy} \mathrm{arcsec}^{-2}$. Far right: residuals of the minimum $\chi^{2}$ model, with overplotted contours of \pm 2 and 3 times the root-mean-square intensity. There do not appear to be any significant structures in the residuals, indicating that the model explains the data well. 
$r_{1}=101_{-38}^{+72}$ au, $r_{2}=318_{-54}^{+43}$ au, $I=16_{-11}^{+11} \mathrm{deg}$, and $\theta=5_{-46}^{+38} \mathrm{deg}$. As expected, $F_{\text {unres }}$ and $F_{\text {res }}$ are anticorrelated, with their sum being well constrained to $118_{-3}^{+3} \mathrm{mJy}$. Similarly, $r_{1}$ is correlated with $F_{\text {unres }}$; for a greater amount of unresolved flux, the resolved disc needs to contribute less flux close to the star. Including the stellar flux, and applying the correction factor of 1.16 for lost flux as outlined above, our modelling implies a total flux of $144 \pm 3 \mathrm{mJy}$, which is just consistent with the $154 \pm 7 \mathrm{mJy}$ we measured using aperture photometry for the purposes of SED fitting. We show the unconvolved image corresponding to the best-fitting (i.e. minimum $\chi^{2}$ ) model in the centre right-hand panel of Fig. A1, and the resulting residuals in the rightmost panel; the residuals show that this model explains the data well. Its parameters are $F_{\text {unres }}=3 \mathrm{mJy}$, $F_{\text {res }}=115 \mathrm{mJy}, r_{1}=60 \mathrm{au}, r_{2}=345 \mathrm{au}, I=22 \mathrm{deg}$, and $\theta=15 \mathrm{deg}$. The best-fitting values of $F_{\text {unres }}, F_{\text {res }}$, and $r_{1}$ are notably different from their medians, which is a result of the correlations between the parameters discussed above. While the stellar orbit of HD 95698 is not well known and we are thus unable to test for misalignment between the disc and the binary plane, the radii derived from image modelling are useful for comparison with the disc size derived from SED fitting (see Section 4.3).

This paper has been typeset from a $\mathrm{T}_{\mathrm{E}} \mathrm{X} / \mathrm{L} \mathrm{T}_{\mathrm{E}} \mathrm{X}$ file prepared by the author. 